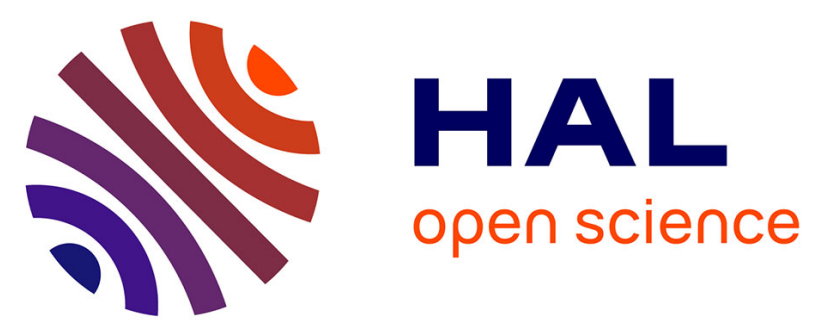

\title{
STRUCTURAL EVIDENCE FOR THE FUNCTIONAL IMPORTANCE OF THE HEME DOMAIN MOBILITY IN FLAVOCYTOCHROME b2
}

K. H. Diêp Lê, Florence Lederer, Béatrice Golinelli-Pimpaneau

\section{- To cite this version:}

K. H. Diêp Lê, Florence Lederer, Béatrice Golinelli-Pimpaneau. STRUCTURAL EVIDENCE FOR THE FUNCTIONAL IMPORTANCE OF THE HEME DOMAIN MOBILITY

IN FLAVOCYTOCHROME b2. Journal of Molecular Biology, 2010, 400 (3), pp.518-530. 10.1016/j.jmb.2010.05.035 . hal-03282526

\section{HAL Id: hal-03282526 \\ https://hal.science/hal-03282526}

Submitted on 9 Jul 2021

HAL is a multi-disciplinary open access archive for the deposit and dissemination of scientific research documents, whether they are published or not. The documents may come from teaching and research institutions in France or abroad, or from public or private research centers.
L'archive ouverte pluridisciplinaire HAL, est destinée au dépôt et à la diffusion de documents scientifiques de niveau recherche, publiés ou non, émanant des établissements d'enseignement et de recherche français ou étrangers, des laboratoires publics ou privés. 


\section{STRUCTURAL EVIDENCE FOR THE FUNCTIONAL IMPORTANCE OF THE HEME DOMAIN MOBILITY IN FLAVOCYTOCHROME $b_{2}$}

\section{K. H. Diêp Lê ${ }^{1}$, Florence Lederer ${ }^{1,2}$ and Béatrice Golinelli-Pimpaneau ${ }^{1}$}

From ${ }^{1}$ Laboratoire d'Enzymologie et Biochimie Structurales, CNRS, 1 avenue de la Terrasse, 91198 Gif-sur-Yvette, France, ${ }^{2}$ Present address: Laboratoire de Chimie Physique, CNRS, Université Paris XI, 91405 Orsay, France

Running head : Heme domain mobility in flavocytochrome $b_{2}$

Address correspondence to: Béatrice Golinelli-Pimpaneau, Laboratoire d'Enzymologie et Biochimie Structurales, CNRS, 1 avenue de la Terrasse, 91198 Gif-sur-Yvette Cedex, France

Tel: 331698242 35; fax: 331698231 29; E-mail: beatrice.golinelli@lebs.cnrs-gif.fr

Yeast flavocytochrome $b_{2}$ (Fcb2), is an L-lactate:cytochrome $c$ oxidoreductase in the mitochondrial intermembrane space participating in cellular respiration. Each enzyme subunit consists of a cytochrome $b_{5}$-like heme domain and a flavodehydrogenase (FDH) domain. In the Fcb2 crystal structure, the heme domain is mobile relative to the tetrameric FDH core in one out of two subunits. The monoclonal antibody B2B4, elicited against the holoenzyme, recognizes only the native heme domain in the holoenzyme. When bound, it suppresses the intramolecular electron transfer from flavin to heme $b_{2}$, hence cytochrome $c$ reduction. We report here the crystal structure of the heme domain in complex with the Fab at $2.7 \AA$ resolution. The Fab epitope on the heme domain includes the two exposed propionate groups of the heme, which are hidden in the interface between the domains in the complete subunit. The structure discloses an unexpected plasticity of Fcb2 in the neighborhood of the heme cavity, in which the heme has rotated. The epitope overlaps with the docking area of the FDH domain onto the heme domain, indicating that the antibody displaces the heme domain in a movement of large amplitude. We suggest that the binding sites on the heme domain of cytochrome $c$ and of the FDH domain also overlap and therefore that cytochrome $c$ binding also requires the heme domain to move away from the FDH domain, so as to allow electron transfer between the two hemes. Based on 
this hypothesis, we propose a possible model of the Fcb2-cytochrome $c$ complex. Interestingly, this model shares similarity with that of the cytochrome $b_{5} \cdot$ cytochrome $c$ complex, in which cytochrome $c$ binds to the surface around the exposed heme edge of cytochrome $b_{5}$. The present results therefore support the idea that the heme domain mobility is an inherent component of the Fcb2 functioning.

Keywords: Flavocytochrome $b_{2}$, Electron transfer, Domain mobility, Monoclonal antibody, cytochrome $c$.

\section{Introduction}

Flavocytochrome $b_{2}(\mathrm{Fcb} 2)(\mathrm{EC} 1.1 .2 .3$.$) is a natural soluble complex of electron transfer proteins that$ has attracted much attention as a model for more complex membranous systems. Fcb2 is an L-lactate dehydrogenase present in the intermembrane space of yeast mitochondria participating in cellular respiration. It enables yeast to grow on L-lactate as sole carbon source, by mediating electron transfer to oxygen via cytochrome $c$ and cytochrome $c$ oxidase. Each subunit of the tetrameric enzyme is composed of an N-terminal heme-binding domain carrying protoporphyrin IX with a cytochrome $b_{5^{-}}$ fold (residues 1 to 99) and a C-terminal flavodehydrogenase (FDH) domain, which binds FMN at the C-terminal end of a $\beta_{8} \alpha_{8}$ barrel (residues 100 to 511$)^{1}$. The core of the enzyme structure is formed by the packing of four FDH domains while the heme domains lie at the periphery. The asymmetric unit of the Fcb2 crystal structure contains a dimer. The heme domain is observed in only one of the two subunits, indicating it is mobile. NMR data show it is also mobile in solution ${ }^{2}$. In the complete subunit, the relative orientation of the heme and FDH domains appears suitable for intrasubunit electron transfer ${ }^{1,3}$.

During the catalytic cycle, L-lactate donates two electrons to FMN (F) (step a in Scheme 1). The reduced FMN (F:) transfers the electrons one by one to protoheme IX $(\mathrm{H})$ in the same subunit (steps b and d); heme $b_{2}$ is reoxidized by the physiological electron acceptor cytochrome $c$ (steps c and e) ${ }^{4}$. Experimental evidence about the docking area of cytochrome $c$ on the enzyme is lacking. The non physiological acceptor ferricyanide normally accepts electrons both from heme and the flavin 
semiquinone $(\mathrm{F} \bullet)$. It can accept electrons from reduced flavin $(\mathrm{F}:)$ only when the flavin to heme intramolecular transfer (step b) is slowed down ${ }^{5,6,7}$.

Miles et al. ${ }^{8}$ have obtained a monoclonal antibody (B2B4), raised against the Fcb2 tetramer, that prevents electron transfer between FMN and protoheme IX and hence inhibits cytochrome $c$ reduction, but has no effect on flavin reduction by L-lactate (Scheme 1, step a) and reoxidation by ferricyanide. Antibody B2B4 recognizes the native heme domain as well as tetrameric Fcb2 with a $K_{d}$ lower than $10^{-7} \mathrm{M}$ and has no affinity for the recombinant FDH domain. The location of the antibody epitope on Fcb2 was previously analyzed by site-directed mutagenesis of residues of the heme domain and by chemical modification of the heme propionate groups ${ }^{9}$. Residues around the heme ligand His66, as well as one or both heme propionates, were shown to belong to the surface of interaction between Fcb2 and antibody B2B4. Most of these residues are buried in the crystal in the interface between the flavin and heme domains. This suggested that the antibody can displace the heme domain from the FDH domain and, once bound, acts as a wedge between the domains, thus maintaining the prosthetic groups in a relative orientation improper for interdomain electron transfer.

In order to further elucidate the structural basis for the uncoupling between the flavin and heme domains by the antibody, we have solved the crystal structure of the antibody Fab fragment in complex with the isolated Fcb2 heme domain at $2.7 \AA$ resolution. The structure confirms that the antibody displaces the heme domain from the FDH domain and highlights the importance of heme domain mobility in the enzyme functioning. In view of the present results, we have used the docking program $H A D D O C K$ to propose a new model for the Fcb2 cytochrome $c$ complex, in which the heme domain has moved away from the FDH domains in order to interact with cytochrome $c$.

\section{Results}

Amino acid sequence of Fab B2B4. The heavy and light chains of antibody B2B4 were shown previously to belong to the IgG1 and $\lambda$ classes, respectively ${ }^{8}$. Amino-terminal sequencing of the whole immunoglobulin yielded the amino acid sequence of the heavy chain only, up to residue 29 with 
gaps at positions 22 and 28. Amino-terminal sequencing of the individual chains separated by SDS gel electrophoresis confirmed that the light chain had a blocked $\mathrm{N}$ terminus. The cDNA of the Fab was cloned and sequenced using consensus sequences for the two chains. The deduced protein sequences are given in Fig. S1A and S1B for the light and heavy chains, respectively. For the mature 213 residue-long light chain, the expected amino acid sequence begins at position 20 of the deduced sequence with a glutamine, in agreement with the expectation that the blocked $\mathrm{N}$ terminus is a pyroglutamic acid. For the 225 residue-long mature heavy chain, the nucleic acid sequence confirms the results of $\mathrm{N}$ terminal sequencing.

Overview of the crystal structure of the heme domain in complex with Fab B2B4. The structure of the heme domain.Fab complex was determined at $2.7 \AA$ resolution by molecular replacement (Table 1). The crystal belongs to space group $\mathrm{P} 2_{1}$ and contains two molecules in the asymmetric unit that are related by a $179.3^{\circ}$ rotation and a $36.1 \AA$ translation with an overall rmsd for $\mathrm{C} \alpha$ atoms of $0.38 \AA$ for the heme domains and $0.25 \AA$ for the Fabs. The two molecules have nearly identical structures and very similar $B$ factors. The head to tail arrangement of the two complexes (Fig. 1A) results in the heme domain being sandwiched between the combining site of one Fab and the constant light chain of the other one. Region H128-H133 in $\mathrm{CH}_{1}$, which belongs to a loop that is disordered in most Fab crystal structures, was not modeled. The $B$-factors of the heme and heme domains in complex with the Fab (40.5 $\AA^{2}$ and $45.3 \AA^{2}$, respectively) are similar to those of the native $\left(33 \AA^{2} \text { and } 52 \AA^{2}\right)^{1}$ or recombinant $\left(41 \AA^{2}\right.$ and $\left.63 \AA^{2}\right)$ Fcb2 ${ }^{10}$. As in these two proteins, residues 6 and 7 have relatively high $B$-factors, indicating they are poorly ordered. Residues 98 to 100 of the heme domain, which were not observed in the electron density, were not modeled.

Interactions of the heme domain with the antibody combining site. The heme domain and the Fab interact tightly via complementary surfaces with a total contact area on the heme domain of $1456 \AA^{2}$ (Fig. 1C). The heme domain and protoheme IX bury $13 \%$ and $48 \%$ of their accessible surface upon Fab binding, respectively. Nine H-bonds as well as extensive hydrophobic interactions, mainly with aromatic residues of the Fab hypervariable loops, contribute to the binding between the two proteins but there are no direct ionic interactions (Fig. 1B, Table 2). The epitope consists of Asn30, residues 63 to 65 (Glu-Pro-Leu) and 67 to 70 (Ala-Pro-Asn-Val), Asp72, Tyr74 and the two heme propionate 
groups, which are in van der Waals contacts with loop H2. Furthermore, the CA-propionate group is involved in a hydrogen bonding interaction with SerH52, whereas the CD-propionate group interacts with a lysine in the constant light chain of the second Fab molecule. This interaction is probably the result of crystal packing. Altogether, all hypervariable loops except the L2 loop provide interactions with the antigen.

Site-directed mutagenesis of Fcb2 previously showed that the mutations E63K, A67Q or L and N69K in the neighborhood of the second heme ligand His66 decreased the affinity for antibody B2B4 by more than three orders of magnitude ${ }^{9}$. From the crystal structure, it is clear that these mutations, which introduce bulk and charge, must disrupt the interactions with the antibody. The P64Q or R, L65A and V70M mutations led to 2- to 8-fold decreases in affinity for the antibody. The relatively loose interactions between residues 64 or 70 and the Fab (van der Waals but no H-bonding interactions) are in agreement with the weaker effects of their mutations to the bulky Gln, Arg and Met groups. The L65A mutation disturbs the van der Waals contacts but probably not the H-bond that occurs through the main chain carbonyl group. Replacing protoheme IX with its dimethyl ester resulted in an affinity decrease for the Fab of more than one order of magnitude. This suggested that at least one heme propionate belongs to the epitope, which is the case as described above. The D72A, K73A and Y74F mutations in the heme domain had no effect on its affinity for the antibody. The lack of effect of the latter mutation is understandable, since the van der Waals interaction of the phenyl ring can be preserved by the substitution with Phe. The situation is less clear for the mutations at positions 72 and 73 since, according to the structure of the complex, these residues form the edge of the epitope. Asn30 and Pro68 had not been substituted.

Structural comparison between the heme domain in complex with Fab B2B4 and in the holoenzyme. The crystal structures of the heme domain in the native and recombinant holoenzyme refined at $2.4 \AA$ (PDB code $1 \mathrm{FCB})$ and $2.3 \AA$ (PDB code $1 \mathrm{KBI})$ resolution, respectively, are highly similar ${ }^{1,10}$. The Fab-bound heme domain and that in the holoenzyme (1FCB) can be superimposed with an rmsd of $0.96 \AA$ over $97 \mathrm{C} \alpha$ atoms (Fig. 2A). The regions in which the polypeptide chain differs most significantly are the N-terminus (residues 6 to 10), residues 20 to 24 , residues 65 to 74 and the C-terminus (residues 88 to 97). But differences at the $\mathrm{N}$ and $\mathrm{C}$ termini as well as in the 20 to 
24 region are also observed between the two holoenzyme structures. Thus, only the chain modifications from residues 65 to 74 are specific to the complex with the Fab. The Ca largest displacements occur at positions $67(1.8 \AA), 68(1.9 \AA)$ and $72(1.5 \AA)$. The chain displacement results from the interaction of these residues with the Fab. The most striking difference between the heme domain in the complex and the holoenzyme is shown by the heme, which undergoes a rotation, more or less in the same plane, of $20^{\circ}$ and a translation of $0.3 \AA$ (Fig. 2A and B), as indicated by the clearly defined electron density of Protoheme IX (Fig. 2C). This movement does not affect the heme ligation by the histidines. The hydrophobic interactions of the pyrrole rings substituents undergo small modifications, with slight shortening or lengthening of distances to hydrophobic groups that line the heme cavity. The ring B methyl group is displaced by $2.8 \AA$ and loses an interaction with Val70, while the ring D methyl group is displaced by $1.6 \AA$ and loses an interaction with Ile61. The ring B vinyl group loses close contacts with Phe39 and forms new interactions with with Ile29 and Leu36. The other interactions are maintained. The fact that Protoheme IX can adopt significantly different orientations, depending on the interacting protein partner, reveals its dynamic behavior inside the cavity. The alterations in the heme position are concomitant with the movements of residues 65 to 74 and could be the result of the interaction with the antibody.

The binding sites of the FDH domain and antibody B2B4 on the heme domain overlap. Although antibody B2B4 was elicited against intact, tetrameric Fcb2, the Fab affinity for the heme domain is identical within error with that for the holoenzyme ${ }^{8}$. Furthermore, the recombinant FDH domain is not recognized by the antibody, either alone or in the presence of the independently expressed heme domain. Therefore, the epitope should be equally accessible on Fcb2 and on the heme domain alone. However, since several residues defining the epitope (Table 2) are also involved in the recognition of the FDH domain (Fig. 2D, ${ }^{1,3}$ ), binding of the antibody to Fcb2 must involve a movement of the heme domain relative to the FDH domain. Indeed, superposition of the structures of the heme domain in complex with the Fab and in the Fcb2 subunit (Fig. 3A) leads to severe steric clashes, indicating that the Fab cannot bind if the heme domain is docked to the FDH domain as observed in the Fcb2 crystal structure. A minimal movement, for example around the 98-99 peptide bond, necessary to push aside the heme domains for Fab binding is illustrated in Fig. 3B, and the resulting rotation of the heme 
domain relative to the FDH domain in Fig. 3C. Other movements of larger amplitude can also be simulated. The extended overlap of the binding sites of antibody B2B4 and the FDH domain on the heme domain thus constitutes an additional piece of evidence for the mobility of the heme domain and suggests that it can undergo movements of reasonably large amplitude.

Modeling the interaction of the heme domain with cytochrome $c$. Cytochrome $c$ takes electrons exclusively from heme $b_{2}{ }^{5,6}$. Two models were previously proposed for the complex of cytochrome $c$ and Fcb2, in which cytochrome $c$ interacts with both domains ${ }^{11,12}$. In these models, the relative orientation of the two domains observed in the Fcb2 crystal structure was maintained. Since Fcb2 can accommodate a molecule as large as an antibody on its interdomain surface, then it should also be able to accommodate a smaller cytochrome $c$ molecule without any interference by the FDH domain. This idea led us to attempt to model an Fcb2·cytochrome $c$ complex, using the program HADDOCK, based on the idea that the epitope of antibody B2B4 may be part of the interaction area with cytochrome $c$. $H A D D O C K$ uses information from identified or predicted protein interfaces for defining unambiguous or ambiguous interaction restraints (AIRs) to drive the docking process, which consists of three stages: randomization of orientations and rigid body energy minimization, semi-rigid simulated annealing in torsion angle space and final refinement in Cartesian space ${ }^{13}, 14$. We first modeled the interaction of yeast iso-1-cytochrome $c$ and the isolated Fcb2 heme domain. For this modeling, we used the residues defining the Fab epitope as active residues (directly involved in the interaction) in unambiguous interaction restraints. In the absence of mutagenesis data to define cytochrome $c$ residues involved in the interaction with Fcb2, we used the comparison of the predicted interactions surfaces of eukaryotic cytochromes $c$ on mammalian cytochromes $b_{5}$ (Fig. 5 in ${ }^{15}$ ), determined using site-directed mutagenesis or NMR data coupled with modeling ${ }^{15,16,17}$. It appears that cytochrome $c$ residues 13,16 , 72,86 and 87 (mammalian cytochrome $c$ numbering) are involved in the interaction with cytochrome $b_{5}$ in all models. Therefore, these residues were chosen as active residues in the modeling. Two clusters were identified on the basis of pairwise backbone rmsd values using a $2.5 \AA$ cutoff criterion. Their statistical analysis is summarized in Table S2. The first cluster, which contains by far the largest number of structures, has the lowest intermolecular energy and the highest buried surface area. The best solution from this cluster has an intermolecular energy of $-561.5 \mathrm{kcal} \mathrm{mol}^{-1}$, an rmsd 
from the starting structures of the heme domain of Fcb2 and cytochrome $c$ of $0.74 \AA$ and $0.62 \AA$, respectively, and a buried surface area of $1037.9 \AA^{2}$ (Fig. S2A). Such a relatively small interface size is compatible with a relatively weak binding between the two redox partners, which allows an easy dissociation of the complex necessary for efficient electron transfer ${ }^{18}$. The minimal distance between the heme porphyrin rings of heme $b_{2}$ and cytochrome $c$ (methyl groups of rings $\mathrm{A}$ and $\mathrm{C}$, respectively) is $9.4 \AA$, which appears compatible with intermolecular electron transfer.

The modeling of one complete Fcb2 subunit in complex with cytochrome $c$ (Fig. 3D) was carried out by superposing the heme domain in the best solution for docking on cytochrome $c$ (Fig. S2A) and that in the minimal model that would allow binding of the Fab (as shown in Fig. 3B). In the present model, four cytochromes $c$ can be accommodated on the Fcb2 tetramer, with each cytochrome $c$ forming interactions with only one subunit. In spite of early controversies, it appears that in solution the stoichiometry is indeed one heme $c$ per heme $b_{2}$, as reviewed in ${ }^{19}$.

\section{Discussion}

Structural alterations of the heme domain bound to Fab. The crystal structure of the Fab·Fcb2 heme domain complex presented here confirms the outline of the epitope defined by site-directed mutagenesis ${ }^{9}$. Altogether, the epitope is located somewhat asymmetrically on one side of the heme crevice, that corresponding to the second heme ligand His66. Interestingly, the heme domain adopts a conformation different from that observed in the crystal structure of the holoenzyme, with a slight movement of the peptide chain segment encompassing His66, as well as a surprising rotation of the heme in its cavity, without any alteration of the heme ligation. There are other examples in the literature of structural modifications of protein antigens incurred upon binding to antibodies, without speaking of alterations between free and complexed antibodies ${ }^{20}$. Deformations of the antigen can occur in particular when the epitope is in a flexible part of the structure. In the present case, the alterations are observed between two bound forms of the heme domain in two different redox states (reduced in the Fcb2 crystal structure, oxidized in the complex with the Fab). One may wonder if there exists for the Fcb2 heme binding domain a limited number of interconvertible conformations in the 
upper part of the heme cavity, such as that observed in the interaction with the FDH domain and that bound to the Fab. Alternatively, the area surrounding the heme could be highly dynamic, with the antibody and the FDH domain each selecting a different conformation of the heme domain in an induced fit mechanism. While this question may receive experimental answers from future studies of the free heme domain, it immediately raises the problem of the possible functional significance of the observed conformational changes, and this will be discussed below.

Displacement of the heme domain from the FDH domain by the antibody. Strikingly, the epitope encompasses residues, which interact with the FDH domain in the crystal structure of the complete subunit ${ }^{1}$ (Fig. 3A and 2D). The importance of these residues in the flavin to heme electron transfer process has recently been evaluated by site-directed mutagenesis ${ }^{3}$. In particular, the small size of the A67 side chain was found to be critical for electron transfer to take place, hence for productive docking of the heme domain to the FDH domain. It is also critical for antibody binding, since replacing the A67 methyl group by Gln and Leu side chains decreased the antibody affinity by at least 3 orders of magnitude ${ }^{9}$. It is clear therefore that the antibody can bind only when the heme domain is not docked to the FDH domain. Moreover, it can be easily understood that the capping of residue 67 , and those surrounding it, with the bulk of the antibody prevents electron transfer between the prosthetic groups, whatever the mechanism of this transfer, through bond or through space. In the minimal model of Fcb2 in complex with the Fab (Fig. 3B), the shortest distance between the isoalloxazine and heme $b_{2}$ rings is $17.2 \AA$ (between the 7 methyl group and the ring D methyl group, respectively). The distances of the flavin N5 atom with the heme ring A C2 atom and with the iron are $18.7 \AA$ and $20.2 \AA$, respectively, compared to $9.7 \AA$ and $13.9 \AA$ in the Fcb2 crystal structure, respectively. The relative orientation of the two cofactors is different from that in the Fcb2 crystal structure and all the interactions between the domains shown to be important for flavin to heme electron transfer to occur are broken ${ }^{1}$. The crystal structure of the Fab-heme domain complex therefore provides an explanation to the observation that binding of the antibody inhibits the intramolecular electron transfer ${ }^{8}$, especially if one considers that the heme domain mobility may have a larger amplitude than the minimal one modeled in Fig. 3C. The domain displacement shown in Fig. 3C was obtained by a rotation around the 98-99 peptide bond. These residues belong to the 
polypeptide linker joining the two domains, commonly called the hinge region. The crystal structure of the subunit indicates, for residues 85 to 99 , a loose interaction with the heme domain, apart from two hydrogen bonds between the peptide amido and carbonyl groups of Asp57 and the carbonyl and amido groups of Leu92 and Cys94, respectively. In addition, tryptic proteolysis of native Fcb2 yields a domain, which ends at Lys95 and has all the physical properties of the heme domain in the intact enzyme ${ }^{4}$. Thus, the hinge region likely contributes to the domain mobility, an idea also supported by the properties of the variant enzymes in which the hinge length has been manipulated ${ }^{21,22}$.

Interaction between Fcb2 and cytochrome c. The interactions between Fcb2 and cytochrome $c$, its physiological acceptor, have been the object of many studies (reviewed in ${ }^{19}$ ). A $1 / 1$ ionic strengthsensitive complex is formed before the electron transfer process, which is itself independent of ionicstrength. Altogether the results indicated that the electron transfer itself occurred via the same mechanism and the same pathway in the presence or the absence of the FDH domain. The interaction of cytochrome $c$ with its other redox partners is also well known to be sensitive to ionic strength ${ }^{23}$. Crystal structures have been determined for its complex with cytochrome $c$ peroxidase ${ }^{24}$ and the cytochrome $b c_{1}$ complex ${ }^{25,26}$. In these complexes, hydrophobic and van der Waals interactions predominate and the non polar interface is surrounded by oppositely charged residue pairs, which are not in close contact. This situation is now considered to be that prevailing in other transient complexes between redox proteins ${ }^{18,27}$.

It is known that the complexes of redox proteins are difficult to crystallize, because of the weak affinity between the partners hence the short lifetime of the complex. In the absence of crystallographic data, modeling was used to predict the interactions between Fcb2 and cytochrome $c$. A first model of the Fcb2-cytochrome $c$ complex was proposed, which involved interactions of cytochrome $c$ with both Fcb2 domains, a 1/1 stoichiometry and contacts of one cytochrome $c$ with two Fcb2 subunits ${ }^{11}$. After this model was shown not to be consistent with subsequent mutagenesis studies ${ }^{28}$, new attempts to model the complex were guided by the fact that the Glu63 and D72 mutations to Lys decreased the rate of electron transfer between reduced Fcb2 and cytochrome $c^{12}$. Although no one single best configuration was obtained, two representative models were described. Both models involved Glu63 and Asp72 of the heme domain as well Glu237 of the FDH domain and basic residues 
at positions 13, 27 and 79 of horse cytochrome $c$. In this complex, cytochrome $c$ was interacting with only one Fcb2 subunit, straddling its two domains.

Does the structural similarity between the Fcb2 heme domain and cytochrome $b_{5}$ extend to the the electron transfer function? The Fcb2 heme domain is homologous to cytochrome $b_{5}$, with $29 \%$ sequence identity and a highly conserved fold ${ }^{1,29}$. The interaction between cytochrome $b_{5}$ and cytochrome $c$ has been extensively studied after an initial model of the complex was proposed by Salemme ${ }^{30}$ (for reviews see $23,27,31,32$ ). Their interaction has been shown by a number of methods to be ionic strength-dependent and highly dynamic. No crystal structure of the cytochrome $b_{5}$. cytochrome $c$ complex is available, but NMR studies, using cytochromes from several species, have provided evidence that the two proteins interact essentially via residues around the solvent-exposed heme edges ${ }^{15,17,33}$. Moreover, the interactions of both cytochrome $c$ and cytochrome $b_{5}$ with their other redox partners also appear to involve this area $24,26,34,35$.

Despite this information and the known mobility of the Fcb2 heme domain, only one group suggested that the heme $b_{2}$ domain interface with the FDH domain could also take part in the interaction with cytochrome $c^{36}$. This hypothesis is supported by the consideration that, in the native Fcb2 subunit crystal structure, the interface between the domains is characteristic of transient complexes, with a small buried surface area $\left(850 \AA^{2}\right)$ and a prevalence of hydrophobic interactions ${ }^{1,3}$. The hypothesis that the heme domain surface around the exposed heme edge interacts both with the FDH domain and cytochrome $c$ is now also supported by the present structure of the Fab.heme domain complex. It may appear in contradiction with the fact that ELISA tests failed to detect a competition between cytochrome $c$ and the Fab for Fcb2 binding, which suggests that cytochrome $c$ and the Fab bind to different sites on the heme domain ${ }^{8}$. Yet, since the dissociation constants of the Fab and of cytochrome $c$ for Fcb2 differ by three orders of magnitude (due to the expected high cytochrome $c$ dissociation rate necessary for turnover) ${ }^{19,28}$, cytochrome $c$ may not have been able to displace the Fab.

We thus decided to use the program $H A D D O C K$ for modeling possible complexes between the heme domain and yeast iso- 1 cytochrome $c$. In all calculations, the same cytochrome $c$ side chains were used as active residues. The best model (Table S2) was obtained with the structure of the heme domain 
from the complex with the Fab, assuming that the epitope residues were involved in the interaction with cytochrome $c$. In this model, yeast iso-1-cytochrome $c$ occupies asymmetrically the side of the heme $b_{2}$ crevice corresponding to the epitope. Besides van der Waals interactions, it forms ionic bonds with Glu63 and Asp72, in agreement with site-directed mutagenesis results ${ }^{12}$, as well as with the two heme propionates. The iron-iron distance of $18.5 \AA$ is close to the value of $17.4 \AA$ observed in the complex of yeast iso-1-cytochrome $c$ with the cytochrome $b c_{1}$ complex ${ }^{25}$ and the inter planar angle of the heme groups is $168^{\circ}$. This model of the Fcb2.cytochrome $c$ complex is similar to those of the cytochrome $b_{5}$.cytochrome $c$ complex ${ }^{15,17}$. Indeed, in addition to $\operatorname{Arg} 13, \operatorname{Gln} 16$, Lys72, Lys86 and Lys87, which were used as active residues to guide the docking, Asn70, Phe82 and Gly83 of yeast iso1-cytochrome $c$ were found to belong to the interface with Fcb2. All these residues were also predicted to interact with cytochrome $b_{5}$. Therefore, although the electrostatic pairing between residues is different, the interaction of cytochrome $c$ with Fcb2 and cytochrome $b_{5}$ is similar in the models calculated with different constraints, originating from different experimental methods, such as NMR or epitope mapping,

One essential difference between the present model and that proposed by Short et al. ${ }^{12}$ for the Fcb2 complex with horse cytochrome $c$ is that our model requires that the heme domain moves relative to the FDH domain in order to interact with cytochrome $c$. This entails in particular a participation of the Fcb2 heme propionate carboxylates in the interaction, while these are hidden in the interface with the FDH domain in the holoenzyme crystal structure. The present most stable model may not exactly describe the interactions that enable electron transfer. It should possibly be considered as an approximation of an encounter complex ${ }^{18,27}$, in which a series of small reorientations could promote the interactions required for electron transfer between the hemes. Indeed, it is thought that the interaction of cytochrome $b_{5}$ with cytochrome $c$ results in a flexible association complex that samples alternative interheme geometries and molecular orientations ${ }^{15,17,37}$.

While future studies are required for improving the model, experimental evidence already exists that is compatible with it, such as the lowering of the electron transfer rate between the Glu63Lys and Asp72Lys Fcb2 variants and cytochrome $c^{12}$. Moreover, Capeillère-Blandin showed that the electron transfer between the Fcb2 heme domain and cytochrome $c$ occurs via the same mechanism and the 
same pathway in the presence or the absence of the FDH domain ${ }^{38}$. This conclusion supports the idea that cytochrome $c$ binds when the heme domain has moved away from the FDH domain.

Finally, mobility of the heme domain may also be required for L-lactate to bind to the active site. Indeed, in the structure of the complete Fcb2 subunit ${ }^{1}$, Tyr143 in the FDH domain forms a hydrogen bond with a heme propionate, which appears to facilitate the interdomain electron transfer ${ }^{39}$. Since this residue also plays a role in L-lactate binding, competition between the substrate carboxylate and the heme propionate for Tyr143 may likely correlate with a movement of the heme domain ${ }^{7}$.

Is the heme domain flexibility functionally important? When the modeling was done using the structure of the heme domain in the holoenzyme (PDB code 1FCB) rather than its structure in complex with the Fab, the calculated complex presented a different relative orientation of the two cytochromes and was less stable. This relative orientation of the heme domain and cytochrome $c$ is mainly due to the different heme orientations in the two Fcb2 heme domain structures. These observations raise the question of the possible functional importance of the flexibility of the Fcb2 heme domain. Does cytochrome $c$ bind to the heme $b_{2}$ domain in a conformation different from that recognized by the FDH domain? Could it bind to the same heme domain conformation as the Fab or still to another one? Does electron transfer between the two proteins require the rotation of heme $b_{2}$ in its cavity? Further experimental work is needed to answer these questions.

\section{Conclusion}

The present experimental results provide concrete evidence for an important degree of freedom of the Fcb2 heme domain relative to the FDH domain, to which it is attached by a covalent bond and a probably flexible linker. In view of all the evidence in the literature, we suggest that in solution the heme domain is constantly sampling space and interacts alternatively with the FDH domain, and cytochrome $c$ when present. The interactions that are favorable for electron transfer implicate in both cases the participation of a number of residues around the exposed heme edge as well as one or both heme propionates. This proposal is strongly supported by a comparison of Fcb2 with a number of redox enzymes, which require large domain movements for the interaction of two or more electron 
transfer partners. Such well characterized systems include a series of homologous diflavin reductases, either isolated ones such as cytochrome P450 reductase ${ }^{40,41,42}$ or included in more complex structures, such as cytochrome P450 BM3 ${ }^{43}$, nitric oxide synthase ${ }^{44,45}$ and sulfite reductase ${ }^{46}$. Electron tranferring flavoprotein (ETF) is another example with a mobile flavodoxin-like domain ${ }^{47,}{ }^{48}$. In cytochrome $b c_{1}$, the iron-sulfur cluster-containing domain is endowed with intracomplex mobility ${ }^{49}$. In the family of cytochrome $b_{5}$ homologues, microsomal cytochrome $b_{5}$ itself is connected to its transmembrane segment by a flexible linker: the latter enables cytochrome $b_{5}$ to interact alternatively with its reductases and its diverse electron acceptors, all of them attached to the endoplasmic reticulum 32, 50. Sulfite oxidase is the system with most similarity to $\mathrm{Fcb} 2$, in that the $b_{5}$-like heme domain is covalently attached to its reductase, a molybdopterin domain, and transfers electrons to cytochrome $c$. In this case, the crystal structure of the chicken liver enzyme is not considered as that of an electron transfer-competent complex, and the flexible peptide loop must allow the orientation changes necessary for shuttling electrons between the molybdopterin domain and cytochrome $c^{51,52,53}$. In all structural and modeling studies with cytochrome $b_{5}$ mentioned above, the surface that interacts with electron acceptors is that around the exposed heme edge. Thus far, Fcb2 is the only system for which both crystallographic and functional details exist concerning the electron transfer-competent interaction of a $b_{5}$-like cytochrome with its reductase, an interaction which again implicates the area around the exposed heme edge ${ }^{1,3}$. Cocrystallization of Fcb2 and cytochrome $c$ remains therefore a challenging goal.

\section{Material and methods}

Proteins. The production and purification of the B2B4 monoclonal antibody and the generation of the Fab fragment by proteolysis with papain were described in ${ }^{8}$. The recombinant $\mathrm{Fcb} 2$ heme domain, encompassing residues 6 to 100 , was produced according to ${ }^{54}$.

Amino acid and nucleotide sequence determination. N-terminal amino acid sequencing was carried out with an Applied Biosystems 476A microsequencer. The antibody mRNA was prepared from 2.10 ascites cells using the QuickPrep mRNA Purification Kit (Pharmacia Biotech). The mRNA (1 $\mu \mathrm{g})$ was reverse-transcribed using the First-Strand cDNA Synthesis Kit (Pharmacia Biotech). The cDNA was 
amplified by the polymerase chain reaction (PCR), using $P f u$ polymerase and the first four oligonucleotides given in Table 4 as primers. The sequence was determined with an ABI Prism sequencer using all the primers given in Table 4, following the instructions of the dRhodamine Terminator Cycle Sequencing Ready Reaction Kit (PE Applied Biosystems).

Crystallization and structure determination. Crystals were grown at $18{ }^{\circ} \mathrm{C}$ in hanging-drops by vapor diffusion. $1 \mu 1$ of the 1:1 heme domain.Fab complex $\left(10.2 \mathrm{mg} \mathrm{ml}^{-1}\right.$ in $0.1 \mathrm{M}$ sodium phosphate $\mathrm{pH}$ 7.2) was mixed with $1 \mu \mathrm{l}$ of a $1 \mathrm{ml}$ reservoir solution (20\% PEG 4000, $0.1 \mathrm{M}$ MES pH 6.5, 0.2 M $\mathrm{MgCl}_{2}$ ). Soaking was performed for a few minutes in a solution of the mother liquor containing $15 \%$ glycerol. Crystals were flash frozen in a cold nitrogen stream at 100K. Diffraction data were collected on beamline ID14EH1 at the European Synchrotron Radiation Facility (Grenoble, France) and processed with MOSFLM and SCALA ${ }^{55,56}$. Table 1 summarizes the data collection and processing statistics. Molecular replacement with $A M o R e^{57}$, using the structure of a single chain variable domain Fv Se155-4 as a model (PDB code 1MFA) ${ }^{58}$, allowed to position the two Fab molecules. After partial refinement of the Fab structures, further molecular replacement with Phaser ${ }^{59}$ using the Fcb2 heme domain as model (PDB code 1FCB) ${ }^{1}$ successfully positioned one of the two heme domains. The other heme domain was generated by using the NCS symmetry of the crystal. Refinement was carried out with $C N S^{60}$ and $R E F M A C 5^{61}$ using manual model adjustments with $O^{62}$. During the refinement, tight NCS restraints for the two light and heavy chains of the Fab and the two heme domains were used until the last cycle of refinement. Superposition of the structures was done with SUPERPK (Alzari, P., personal communication) and calculations of the accessible surface areas with $A S A$.

Calculation of a cytochrome cheme domain complex. The HADDOCK web docking site (http://haddock.chem.uu.nl/) in the expert mode was used with the default parameters except that distance restraints between the hemes and their ligands were included, and the rmsd cutoff for clustering was set to $2.5 \AA$. The docking of yeast iso-1-cytochrome $c$ (pdb code 1YCC) and the Fcb2 heme domain used the following restraints. Ten Fcb2 residues $(30,63,64,65,67,68,69,70,72)$ and 5 residues $(29,60,62,66,73,74)$ were used as active and passive unambiguous intermolecular restraints, respectively. Heme $b_{2}$ was treated as passive, except that the carboxylate oxygens of the CA and CD-propionate groups were treated as active. Residues 13,16, 72, 86 and 87 of cytochrome $c$ 
were treated as active, and heme $c$ as passive. Yeast iso-1-cytochrome $c$ with non methylated Lys72

was used in the modeling but very similar results were obtained with the trimethylated cytochrome (mammalian cytochrome $c$ numbering).

\section{Accession numbers}

Coordinates and structure factors have been deposited in the Protein Data Bank with accession number 3KS0.

\section{Acknowledgements}

We thank Djemel Hamdane for critical reading of the manuscript.

\section{References}

1. Xia, Z. X. \& Mathews, F. S. (1990). Molecular structure of flavocytochrome $b_{2}$ at $2.4 \AA$ resolution. J Mol Biol 212, 837-63.

2. Labeyrie, F., Beloeil, J. C. \& Thomas, M. A. (1988). Evidence by NMR for mobility of the cytochrome domain within flavocytochrome $b_{2}$. Biochim Biophys Acta 953, 134-41.

3. Lê, K. H. D., Boussac, A., Frangioni, B., Léger, C. \& Lederer, F. (2009). Interdomain contacts in flavocytochrome $b_{2}$, a mutational analysis. Biochemistry 48, 10803-9.

4. Lederer, F. (1991). Flavocytochrome $b_{2}$. In Chemistry and Biochemistry of flavoenzymes. (Müller, F., ed.), Vol. 2, pp. 153-242. CRC press, Boca Raton, FL.

5. Iwatsubo, M., Mevel-Ninio, M. \& Labeyrie, F. (1977). Rapid kinetic studies of partial reactions in the heme-free derivative of L-lactate cytochrome $c$ oxidoreductase (flavocytochrome $b_{2}$ ); the flavodehydrogenase function. Biochemistry 16, 3558-66.

6. Balme, A., Brunt, C. E., Pallister, R. L., Chapman, S. K. \& Reid, G. A. (1995). Isolation and characterization of the flavin-binding domain of flavocytochrome $b_{2}$ expressed independently in Escherichia coli. Biochem J 309, 601-5.

7. Rouvière, N., Mayer, M., Tegoni, M., Capeillère-Blandin, C. \& Lederer, F. (1997). Molecular interpretation of inhibition by excess substrate in flavocytochrome $b_{2}$ : a study with wild-type and Y143F mutant enzymes. Biochemistry 36, 7126-35.

8. Miles, C. S., Lederer, F. \& Lê, K. H. D. (1998). Probing intramolecular electron transfer within flavocytochrome $b_{2}$ with a monoclonal antibody. Biochemistry 37, 3440-8.

9. Lê, K. H. D., Mayer, M. \& Lederer, F. (2003). Epitope mapping for the monoclonal antibody that inhibits intramolecular electron transfer in flavocytochrome $b_{2}$. Biochem $J 373,115-23$.

10. Cunane, L. M., Barton, J. D., Chen, Z. W., Welsh, F. E., Chapman, S. K., Reid, G. A. \& Mathews, F. S. (2002). Crystallographic study of the recombinant flavin-binding domain of Baker's yeast flavocytochrome $b_{2}$ : comparison with the intact wild-type enzyme. Biochemistry 41, 4264-72.

11. Tegoni, M., White, S. A., Roussel, A., Mathews, F. S. \& Cambillau, C. (1993). A hypothetical complex between crystalline flavocytochrome $b_{2}$ and cytochrome $c$. Proteins 16, 408-22.

12. Short, D. M., Walkinshaw, M. D., Taylor, P., Reid, G. A. \& Chapman, S. K. (1998). Location of a cytochrome $c$ binding site on the surface of flavocytochrome $b_{2}$. J. Biol. Inorg. Chem. 3, 246-252.

13. Dominguez, C., Boelens, R. \& Bonvin, A. M. (2003). HADDOCK: a protein-protein docking approach based on biochemical or biophysical information. J Am Chem Soc 125, 1731-7. 
14. de Vries, S. J., van Dijk, A. D., Krzeminski, M., van Dijk, M., Thureau, A., Hsu, V., Wassenaar, T. \& Bonvin, A. M. (2007). HADDOCK versus HADDOCK: new features and performance of HADDOCK2.0 on the CAPRI targets. Proteins 69, 726-33.

15. Deep, S., Im, S. C., Zuiderweg, E. R. \& Waskell, L. (2005). Characterization and calculation of a cytochrome $c$-cytochrome $b_{5}$ complex using NMR data. Biochemistry 44, 10654-68.

16. Northrup, S. H., Thomasson, K. A., Miller, C. M., Barker, P. D., Eltis, L. D., Guillemette, J. G., Inglis, S. C. \& Mauk, A. G. (1993). Effects of charged amino acid mutations on the bimolecular kinetics of reduction of yeast iso-1-ferricytochrome $c$ by bovine ferrocytochrome $b_{5}$. Biochemistry 32, 6613-23.

17. Volkov, A. N., Ferrari, D., Worrall, J. A., Bonvin, A. M. \& Ubbink, M. (2005). The orientations of cytochrome $c$ in the highly dynamic complex with cytochrome $b_{5}$ visualized by NMR and docking using HADDOCK. Protein Sci 14, 799-811.

18. Crowley, P. B. \& Carrondo, M. A. (2004). The architecture of the binding site in redox protein complexes: implications for fast dissociation. Proteins 55, 603-12.

19. Capeillère-Blandin, C. (1995). Flavocytochrome $b_{2}$-cytochrome $c$ interactions: the electron transfer reaction revisited. Biochimie 77, 516-30.

20. Sundberg, E. J. \& Mariuzza, R. A. (2003). Molecular recognition in antibody-antigen complexes. Adv Protein Chem 61, 119-60.

21. White, P., Manson, F. D., Brunt, C. E., Chapman, S. K. \& Reid, G. A. (1993). The importance of the interdomain hinge in intramolecular electron transfer in flavocytochrome $b_{2}$. Biochem $J$ 291, 89-94.

22. Sharp, R. E., White, P., Chapman, S. K. \& Reid, G. A. (1994). Role of the interdomain hinge of flavocytochrome $b_{2}$ in intra- and inter-protein electron transfer. Biochemistry 33, 5115-20.

23. Mauk, A. G., Mauk, M. R., Moore, G. R. \& Northrup, S. H. (1995). Experimental and theoretical analysis of the interaction between cytochrome $c$ and cytochrome $b_{5}$. J Bioenerg Biomembr 27, 311-30.

24. Pelletier, H. \& Kraut, J. (1992). Crystal structure of a complex between electron transfer partners, cytochrome $c$ peroxidase and cytochrome $c$. Science 258, 1748-55.

25. Lange, C. \& Hunte, C. (2002). Crystal structure of the yeast cytochrome $b c_{1}$ complex with its bound substrate cytochrome c. Proc Natl Acad Sci U SA 99, 2800-5.

26. Solmaz, S. R. \& Hunte, C. (2008). Structure of Complex III with Bound Cytochrome $c$ in Reduced State and Definition of a Minimal Core Interface for Electron Transfer. J Biol Chem 283, 17542-9.

27. Prudencio, M. \& Ubbink, M. (2004). Transient complexes of redox proteins: structural and dynamic details from NMR studies. J Mol Recognit 17, 524-39.

28. Daff, S., Sharp, R. E., Short, D. M., Bell, C., White, P., Manson, F. D., Reid, G. A. \& Chapman, S. K. (1996). Interaction of cytochrome $c$ with flavocytochrome $b_{2}$. Biochemistry 35, 6351-7.

29. Lederer, F. (1994). The cytochrome $b_{5}$-fold: an adaptable module. Biochimie 76, 674-92.

30. Salemme, F. R. (1976). An hypothetical structure for an intermolecular electron transfer complex of cytochromes $c$ and $b_{5} . J$ Mol Biol 102, 563-8.

31. Guillemette, J. G., Barker, P. D., Eltis, L. D., Lo, T. P., Smith, M., Brayer, G. D. \& Mauk, A. G. (1994). Analysis of the bimolecular reduction of ferricytochrome $c$ by ferrocytochrome $b_{5}$ through mutagenesis and molecular modelling. Biochimie 76, 592-604.

32. Vergères, G. \& Waskell, L. (1995). Cytochrome $b_{5}$, its functions, structure and membrane topology. Biochimie 77, 604-20.

33. Shao, W., Im, S. C., Zuiderweg, E. R. \& Waskell, L. (2003). Mapping the binding interface of the cytochrome $b_{5}$-cytochrome $c$ complex by nuclear magnetic resonance. Biochemistry $\mathbf{4 2}$, 14774-84.

34. Worrall, J. A., Liu, Y., Crowley, P. B., Nocek, J. M., Hoffman, B. M. \& Ubbink, M. (2002). Myoglobin and cytochrome $b_{5}$ : a nuclear magnetic resonance study of a highly dynamic protein complex. Biochemistry 41, 11721-30.

35. Wheeler, K. E., Nocek, J. M., Cull, D. A., Yatsunyk, L. A., Rosenzweig, A. C. \& Hoffman, B. M. (2007). Dynamic docking of cytochrome $b_{5}$ with myoglobin and alpha-hemoglobin: heme- 
neutralization "squares" and the binding of electron-transfer-reactive configurations. $J \mathrm{Am}$ Chem Soc 129, 3906-17.

36. Tollin, G., Hurley, J. K., Hazzard, J. T. \& Meyer, T. E. (1993). Use of laser flash photolysis time-resolved spectrophotometry to investigate interprotein and intraprotein electron transfer mechanisms. Biophys Chem 48, 259-79.

37. Wendoloski, J. J., Matthew, J. B., Weber, P. C. \& Salemme, F. R. (1987). Molecular dynamics of a cytochrome $c$-cytochrome $b_{5}$ electron transfer complex. Science 238, 794-7.

38. Capeillère-Blandin, C. \& Albani, J. (1987). Cytochrome $b_{2}$, an electron carrier between flavocytochrome $b_{2}$ and cytochrome $c$. Rapid kinetic characterization of the electron-transfer parameters with ionic-strength-dependence. Biochem J 245, 159-65.

39. Miles, C. S., Rouvière-Fourmy, N., Lederer, F., Mathews, F. S., Reid, G. A., Black, M. T. \& Chapman, S. K. (1992). Tyr-143 facilitates interdomain electron transfer in flavocytochrome b. Biochem J 285 ( Pt 1), 187-92.

40. Hubbard, P. A., Shen, A. L., Paschke, R., Kasper, C. B. \& Kim, J. J. (2001). NADPHcytochrome P450 oxidoreductase. Structural basis for hydride and electron transfer. J Biol Chem 276, 29163-70.

41. Hamdane, D., Xia, C., Im, S. C., Zhang, H., Kim, J. J. \& Waskell, L. (2009). Structure and function of an NADPH-cytochrome P450 oxidoreductase in an open conformation capable of reducing cytochrome P450. J Biol Chem 284, 11374-84.

42. Ellis, J., Gutierrez, A., Barsukov, I. L., Huang, W. C., Grossmann, J. G. \& Roberts, G. C. (2009). Domain motion in cytochrome P450 reductase: conformational equilibria revealed by NMR and small-angle x-ray scattering. $J$ Biol Chem 284, 36628-37.

43. Sevrioukova, I. F., Li, H., Zhang, H., Peterson, J. A. \& Poulos, T. L. (1999). Structure of a cytochrome P450-redox partner electron-transfer complex. Proc Natl Acad Sci U S A 96, 1863-8.

44. Garcin, E. D., Bruns, C. M., Lloyd, S. J., Hosfield, D. J., Tiso, M., Gachhui, R., Stuehr, D. J., Tainer, J. A. \& Getzoff, E. D. (2004). Structural basis for isozyme-specific regulation of electron transfer in nitric-oxide synthase. J Biol Chem 279, 37918-27.

45. Stuehr, D. J., Tejero, J. \& Haque, M. M. (2009). Structural and mechanistic aspects of flavoproteins: electron transfer through the nitric oxide synthase flavoprotein domain. Febs $J$ 276, 3959-74.

46. Gruez, A., Pignol, D., Zeghouf, M., Covès, J., Fontecave, M., Ferrer, J. L. \& FontecillaCamps, J. C. (2000). Four crystal structures of the $60 \mathrm{kDa}$ flavoprotein monomer of the sulfite reductase indicate a disordered flavodoxin-like module. J Mol Biol 299, 199-212.

47. Leys, D., Basran, J., Talfournier, F., Sutcliffe, M. J. \& Scrutton, N. S. (2003). Extensive conformational sampling in a ternary electron transfer complex. Nat Struct Biol 10, 219-25.

48. Toogood, H. S., Leys, D. \& Scrutton, N. S. (2007). Dynamics driving function: new insights from electron transferring flavoproteins and partner complexes. FEBS J 274, 5481-504.

49. Zhang, Z., Huang, L., Shulmeister, V. M., Chi, Y. I., Kim, K. K., Hung, L. W., Crofts, A. R., Berry, E. A. \& Kim, S. H. (1998). Electron transfer by domain movement in cytochrome $b c_{1}$. Nature 392, 677-84.

50. Clarke, T. A., Im, S. C., Bidwai, A. \& Waskell, L. (2004). The role of the length and sequence of the linker domain of cytochrome $b_{5}$ in stimulating cytochrome P450 $2 \mathrm{~B} 4$ catalysis. $J$ Biol Chem 279, 36809-18.

51. Kisker, C., Schindelin, H., Pacheco, A., Wehbi, W. A., Garrett, R. M., Rajagopalan, K. V., Enemark, J. H. \& Rees, D. C. (1997). Molecular basis of sulfite oxidase deficiency from the structure of sulfite oxidase. Cell 91, 973-83.

52. Feng, C., Tollin, G. \& Enemark, J. H. (2007). Sulfite oxidizing enzymes. Biochim Biophys Acta 1774, 527-39.

53. Johnson-Winters, K., Nordstrom, A. R., Emesh, S., Astashkin, A. V., Rajapakshe, A., Berry, R. E., Tollin, G. \& Enemark, J. H. (2010). Effects of interdomain tether length and flexibility on the kinetics of intramolecular electron transfer in human sulfite oxidase. Biochemistry 49 , 1290-6. 
54. Brunt, C. E., Cox, M. C., Thurgood, A. G., Moore, G. R., Reid, G. A. \& Chapman, S. K. (1992). Isolation and characterization of the cytochrome domain of flavocytochrome $b_{2}$ expressed independently in Escherichia coli. Biochem $J$ 283, 87-90.

55. Leslie, A. G. W. (1992). In Recent changes to the MOSFLM package for processing film and image plate data. Joint CCP4 + ESF-EAMCB Newsletter on Protein Crystallography., Vol. 26.

56. Evans, P. (2006). Scaling and assessment of data quality. Acta Crystallogr D Biol Crystallogr 62, 72-82.

57. Navaza, J. (1994). AMoRe: an automated package for molecular replacement. Acta Cryst. A50, 157-163.

58. Zdanov, A., Li, Y., Bundle, D. R., Deng, S. J., MacKenzie, C. R., Narang, S. A., Young, N. M. \& Cygler, M. (1994). Structure of a single-chain antibody variable domain (Fv) fragment complexed with a carbohydrate antigen at 1.7-Å resolution. Proc Natl Acad Sci U S A 91, 6423-7.

59. McCoy, A. J., Grosse-Kunstleve, R. W., Adams, P. D., Winn, M. D., Storoni, L. C. \& Read, R. J. (2007). Phaser crystallographic software. J Appl Crystallogr 40, 658-674.

60. Brünger, A. T. (2007). Version 1.2 of the Crystallography and NMR system. Nat Protoc 2, 2728-33.

61. Murshudov, G. N., Vagin, A. A. \& Dodson, E. J. (1997). Refinement of macromolecular structures by the maximum-likelihood method. Acta Crystallogr D Biol Crystallogr 53, 24055 .

62. Jones, T. A., Zou, J. Y., Cowan, S. W. \& Kjeldgaard, M. (1991). Improved methods for building protein models in electron density maps and the location of errors in these models. Acta Crystallogr A 47, 110-9. 


\section{Figure legends}

Fig. 1: Structure of the complex between Fab B2B4 and the Fcb2 heme domain. A Stereoview of the asymmetric unit of the heme domain.FabB2B4 complex contains two heme domains ( $\mathrm{C} \alpha$ trace in yellow, protoheme IX in tan stick, iron in red) and two Fab molecules. The C $\alpha$ trace for the light and heavy chains for one Fab molecule are shown in blue and brown, respectively and for the other Fab molecule in cyan. Loops L1, L2, L3, H1, H2 and H3 are shown in magenta to visualize the antibody combining site. In the crystal structure, one heme domain interacts with the combining site of one Fab molecule and with the constant light chain of the other. This figure was drawn with $O^{62}$. B Stereoview of the interactions between residues 63-72 and 30 of the heme domain and the hypervariable regions of Fab B2B4. Cas of hypervariable regions $\mathrm{H} 1, \mathrm{H} 2, \mathrm{H} 3, \mathrm{~L} 1, \mathrm{~L} 2$ and L3 are indicated in red, tan, green, blue, cyan and magenta, respectively. The H-bonds between the heme-domain and antibody are indicated as dashed red lines. $\mathbf{C}$ Molecular surface of the interaction site of the heme domain·FabB2B4 complex. The molecular surface for the light chain, heavy chain and heme domain are shown in transparent blue, green and orange, respectively. Protoheme $\mathrm{X}$ is shown in orange for the buried atoms and yellow for the accessible atoms, the iron atom is shown as a red sphere. The residues belonging to the epitope are indicated as sticks. This figure was drawn with PYMOL (www.pymol.org).

Fig. 2: Comparison of the heme domains from the Fcb2 crystal structure and in complex with the Fab. A Stereoview of the superposition of the heme domains from the Fcb2 crystal structure (PDB code 1FCB) (in cyan, protoheme IX in blue sticks, iron in blue) and in the Fab.heme domain complex (in yellow, protoheme IX in tan sticks, iron in red). B Comparison of the orientations of Protoheme IX in the heme domain.Fab structure (yellow) and in the holoenzyme (blue) after superposition of the C $\alpha$ positions of the heme domains. C Stereo view of the Fobs-Fcalc electron density map of the Protoheme IX region omitting Protoheme IX calculated at the level of 1.5 and 8 standard deviations (green and blue colour, respectively) in the heme domain.Fab structure. D Surface representations of the heme domain interfaces with Fab B2B4 (left) and with the FDH domain (right). The flavin-binding site is as defined (Table 10A in ${ }^{1}$ ). The heme group is orange or green and its accessible atoms are yellow. The residues involved in the 
interaction with the Fab and the FDH domain are indicated as red and blue spheres, respectively. This figure was drawn with PYMOL (www.pymol.org).

Fig. 3: Fab B2B4 binding prevents normal docking of the heme domain onto the FDH domain (Stereoviews). The orientation of the FDH domain is identical in all figures. A Residues 6 to 97 of the heme domain of the Fcb2 structure (PDB code 1FCB) were superimposed on the same residues of the heme domain in the complex with Fab B2B4 (rmsd=0.92 $\AA$ ). The heme domain in the Fcb2 structure is shown in cyan and the FDH domain (residues 98 to 511) in magenta, with FMN in red sticks. The heme domain in the complex with the Fab is shown in yellow with protoheme IX in tan sticks and iron in red. The light and heavy chains of FabB2B4 are shown in blue and brown, respectively. B A possible minimal movement $\left(62.5^{\circ}\right.$ rotation and $0.058 \AA$ translation $)$ of the heme domain relative to the FDH domain in one Fcb2 monomer that would enable the antibody to bind to whole Fcb2. C Superposition of the positions of the Fcb2 heme domains before (in cyan) and after the rotation allowing Fab B2B4 binding (in yellow). D. Model of one Fcb2 monomer in complex with cytochrome $c$. The FDH domain (in magenta, FMN in red sticks) has been added to the model of the heme domain.cytochrome $c$ complex with the orientation relative to the heme domains shown in Fig. 3B, showing that the movement of the heme domain relative to the FDH domain that enables Fab binding is compatible with cytochrome $c$ binding. 
Scheme 1

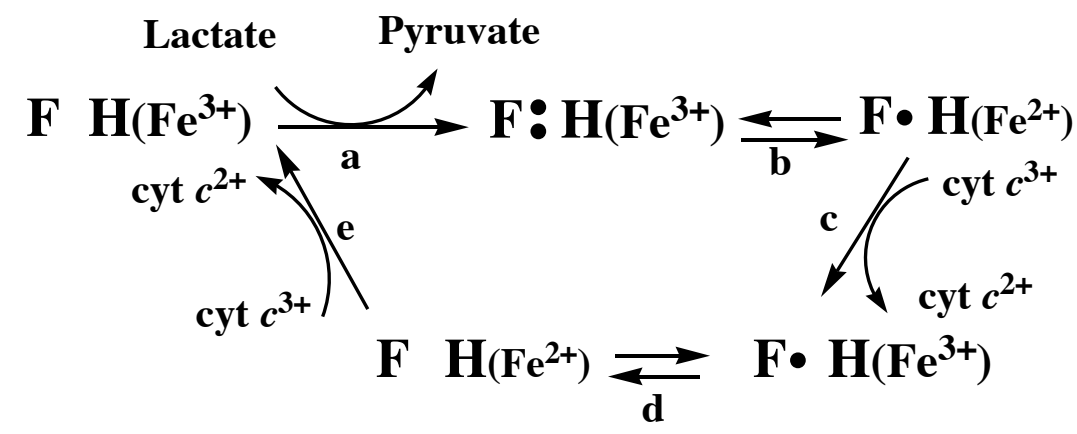


Figure 1

A
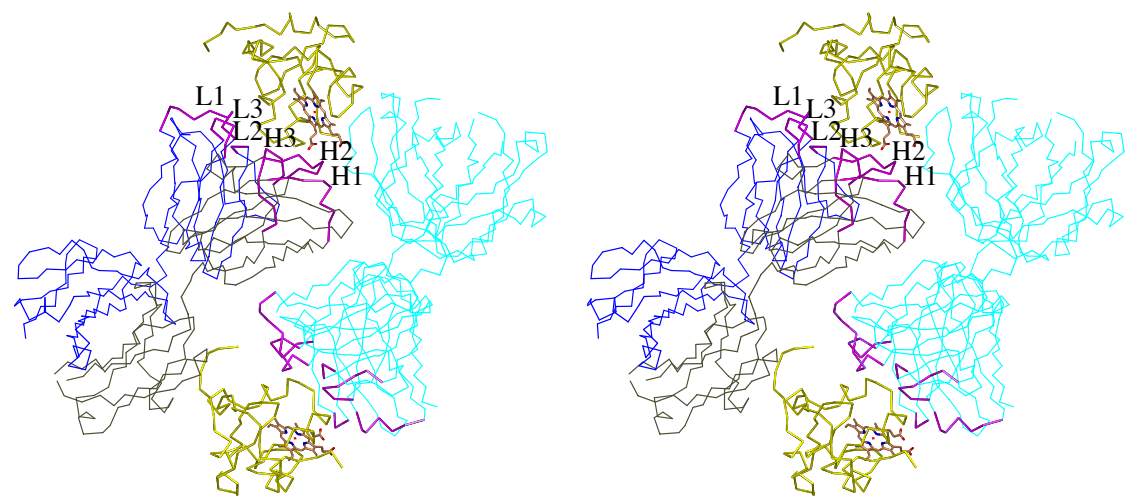

B

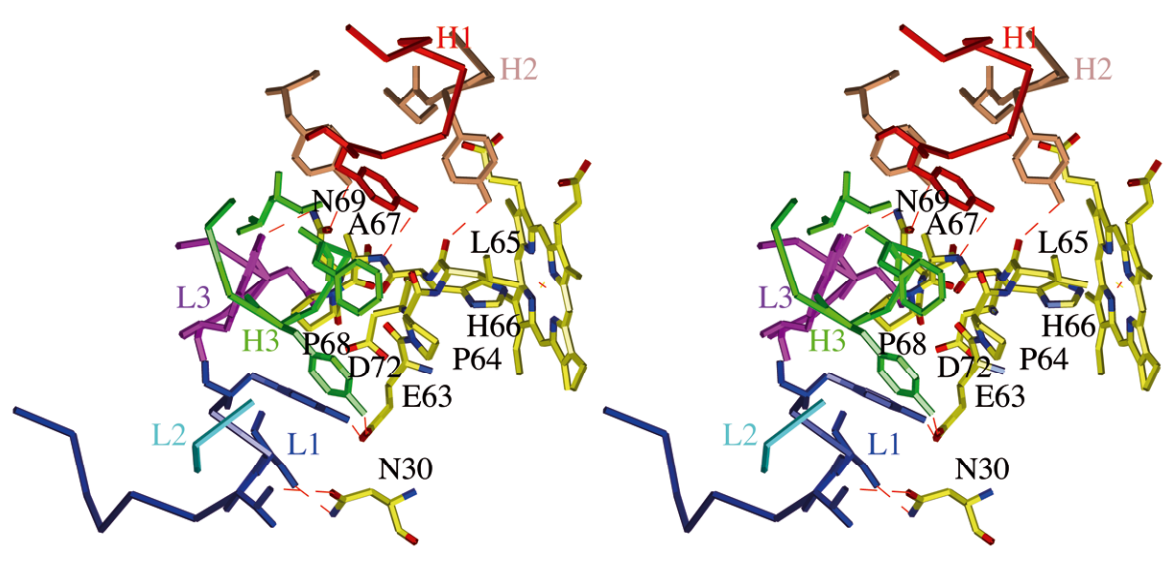

C

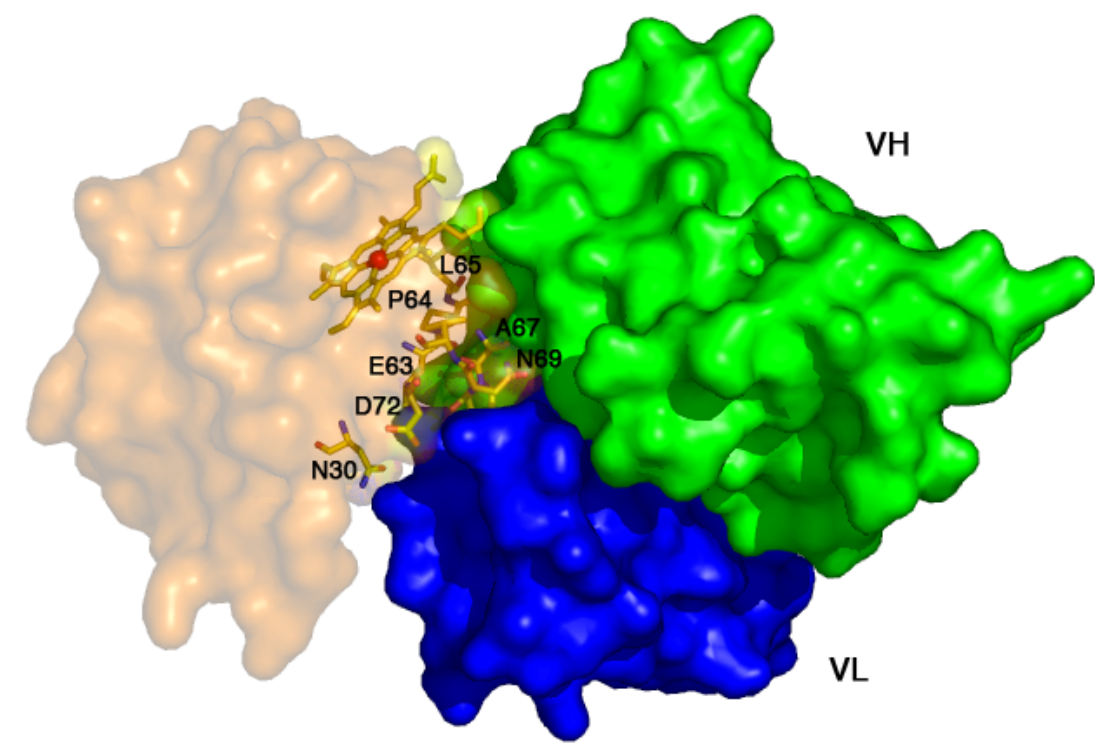


Figure 2

A
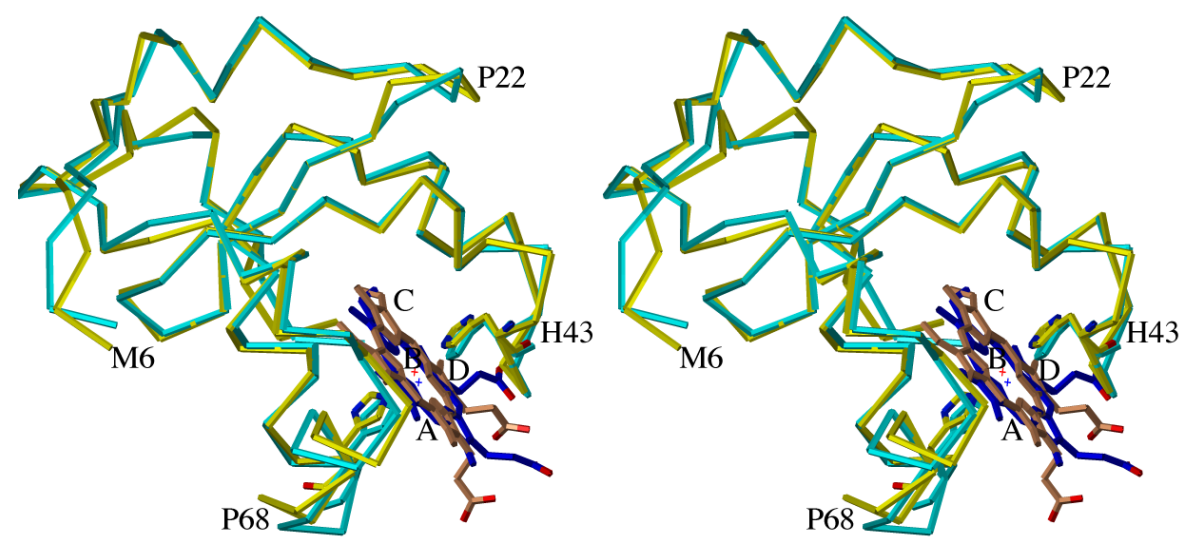

B

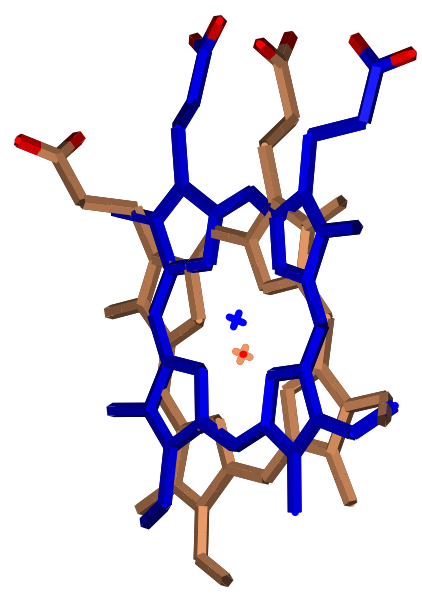

D

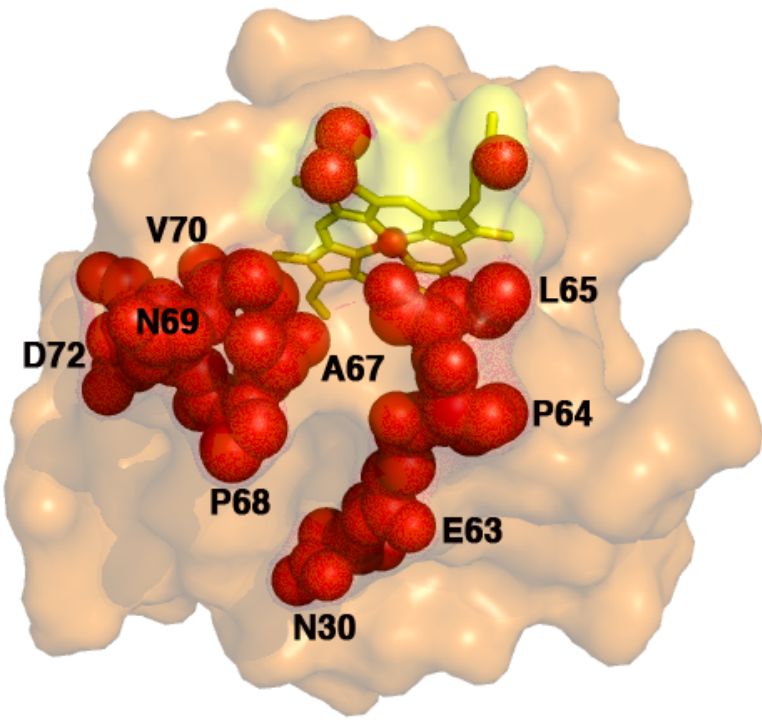

C
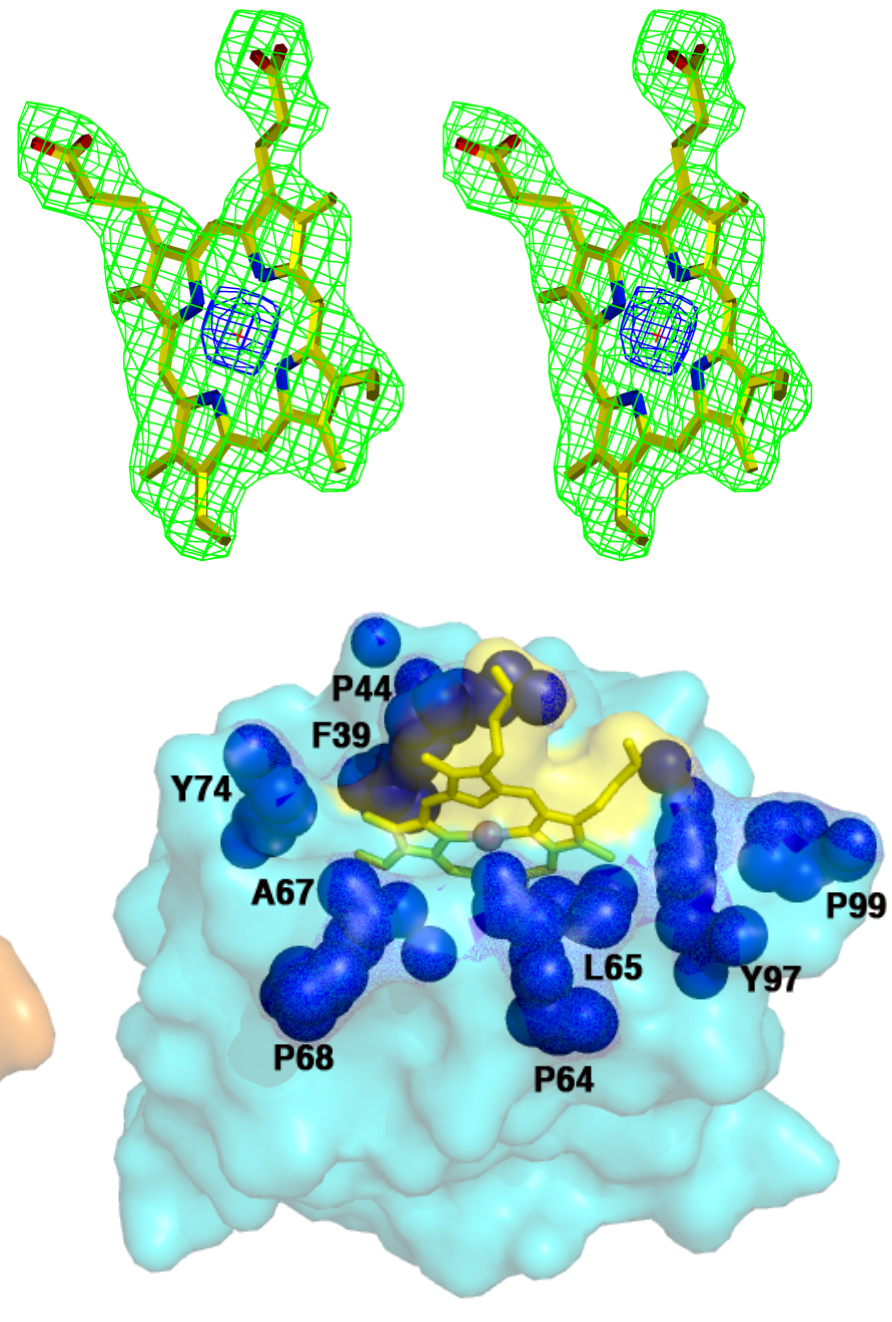
Figure 3
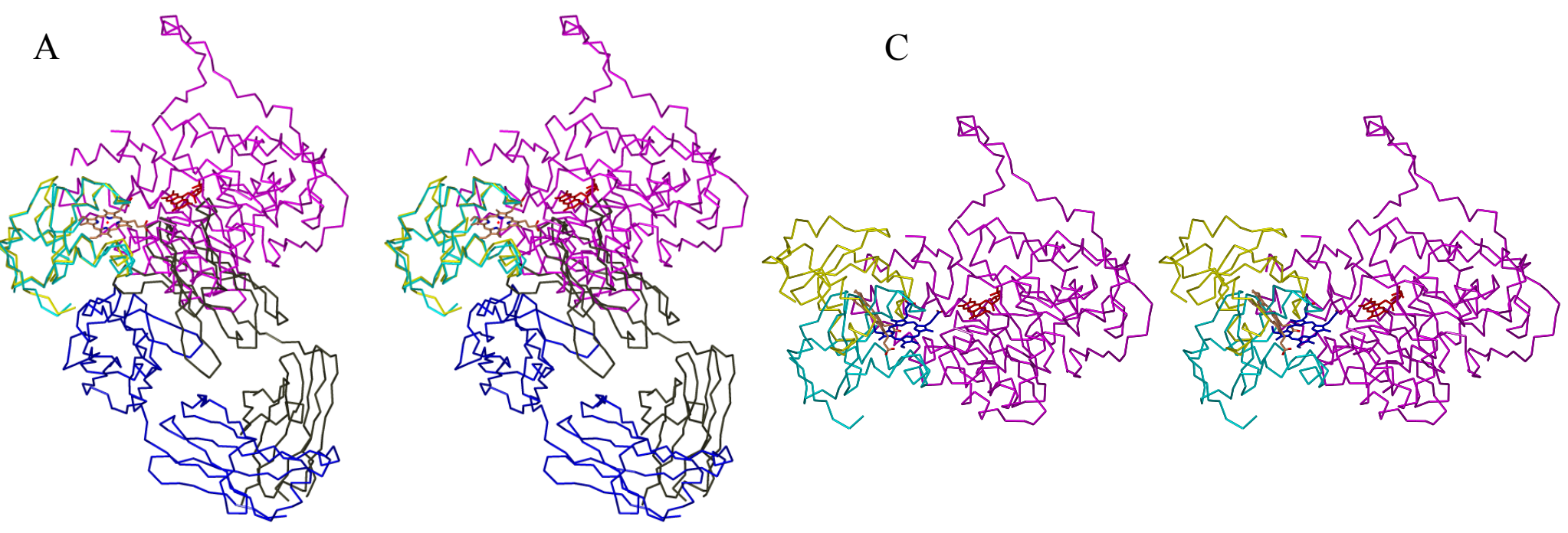

B

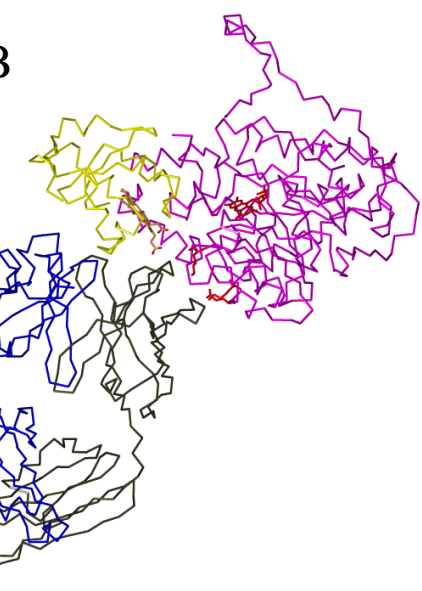

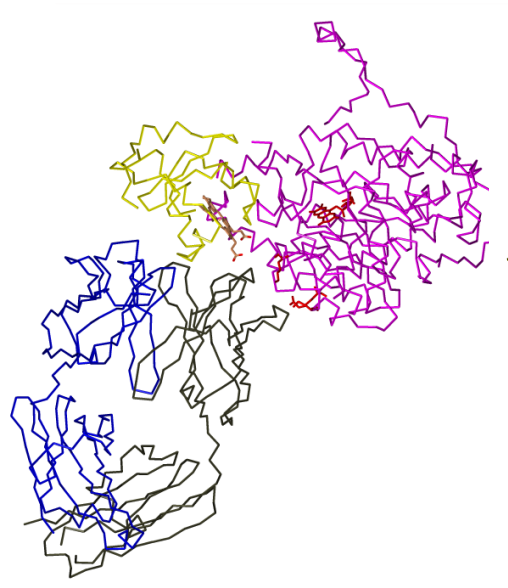

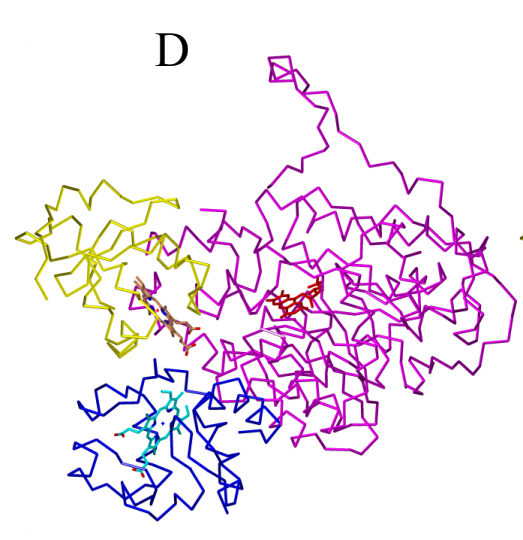

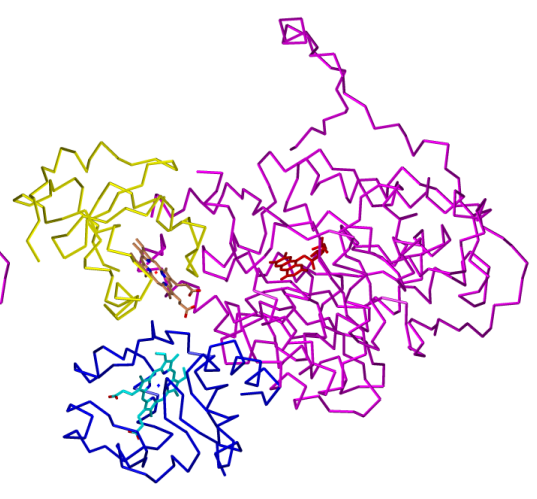


Table 1: Data collection and refinement statistics.

\begin{tabular}{|c|c|}
\hline \multicolumn{2}{|l|}{ Data collection } \\
\hline Space Group & $\mathrm{P} 2_{1}$ \\
\hline Cell dimensions a, b, c $(\AA)$ & $\begin{array}{lll}72.58 & 83.68 & 92.16\end{array}$ \\
\hline$\alpha, \beta, \gamma\left({ }^{\circ}\right)$ & $\begin{array}{lll}90.0 & 96.8 & 90.0\end{array}$ \\
\hline Resolution $(\AA)$ & $25.0-2.7$ \\
\hline (Outer resolution shell) $(\AA)$ & $(2.85-2.7)$ \\
\hline No unique reflections & 29267 \\
\hline $\mathrm{R}_{\text {merge }}$ & $0.16(0.66)$ \\
\hline $\mathrm{I} / \sigma(\mathrm{I})$ & $9.1(1.7)$ \\
\hline Completeness (\%) & $97.0(94.6)$ \\
\hline Redundancy & $3.5(3.3)$ \\
\hline \multicolumn{2}{|l|}{ Refinement } \\
\hline Resolution $(\AA)$ & $25.00-2.7$ \\
\hline (Outer resolution shell) $(\AA)$ & $2.82-2.7$ \\
\hline $\mathrm{R}_{\text {free }}(\%)$ & $28.9(39.3)$ \\
\hline $\mathrm{R}(\%)$ & $21.4(27.6)$ \\
\hline \multicolumn{2}{|l|}{ Average B-factors $\left(\AA^{2}\right)$} \\
\hline $\mathrm{Fab}$ (chains LH/JK) & $44.2 / 43.8$ \\
\hline heme domain (chain $\mathrm{A} / \mathrm{B}$ ) & $45.0 / 45.6$ \\
\hline Heme (chain A/B) & $39.7 / 41.4$ \\
\hline Water & 34.2 \\
\hline \multicolumn{2}{|l|}{ No. atoms } \\
\hline Protein & 7810 \\
\hline heme & 86 \\
\hline Water & 132 \\
\hline \multicolumn{2}{|l|}{ rmsd } \\
\hline Bond lengths $(\AA)$ & 0.015 \\
\hline Bond angles $\left({ }^{\circ}\right)$ & 1.82 \\
\hline \multicolumn{2}{|l|}{ Ramachandran statistics (\%) } \\
\hline Most favored & 82.5 \\
\hline Additionnally allowed & 15.5 \\
\hline Generously allowed & 1.5 \\
\hline Disallowed & 0.5 \\
\hline
\end{tabular}


Table 2. Intermolecular contacts of $4.5 \AA$ or less in the heme $b_{2}$ domain.Fab structure.

\begin{tabular}{|l|l|l|l|}
\hline Heme domain position & Type of interaction & Fab position (loop) & $\mathrm{d}^{1}(\AA)$ \\
\hline Asn30 & van der Waals & ThrL29 (L1) & 3.4 \\
& van der Waals & SerL30 (L1) & 3.6 \\
& van der Waals & TyrL32 (L1) & 3.9 \\
\hline Glu63 OE2 & H-bond & TyrL32 OH (L1) & 2.4 \\
Glu63 OE1 & H-bond & TyrH102 OH (H3) & 3.1 \\
Glu63 O & H-bond & AspH101 O & 2.5 \\
\hline Pro64 & van der Waals & PheH96 (H3) & 3.7 \\
& van der Waals & TyrH99 (H3) & 4.4 \\
\hline Leu65 & van der Waals & ProL155 (CL) ${ }^{2}$ & 4.0 \\
Leu65 O & H-bond & TyrH53 OH (H2) & 3.0 \\
\hline Ala67 N & H-bond & TyrH33 OH (H1) & 3.1 \\
& van der Waals & TyrH50 (H1) & 3.8 \\
\hline Pro68 & van der Waals & TrpL91 (L3) & 3.6 \\
& van der Waals & TyrL32 (L1) & 4.0 \\
\hline Asn69 OD1/ ND2 & H-bond & TyrH50 OH (H2) & 3.0 \\
& van der Waals & AsnL94 O (L3) & 3.5 \\
& van der Waals & TrpL91 (L3) & 3.8 \\
& van der Waals & TyrH58 (H2) & 3.5 \\
\hline Val70 & van der Waals & TyrH50 (H1) & 4.3 \\
& van der Waals & Tyr H33 (H1) & 3.7 \\
\hline Asp72 OD2 & H-bond & AsnL94 OD1 (L3) & 3.3 \\
& H-bond & AsnL94 ND2 (L3) & 3.4 \\
\hline Tyr74 OH & van der Waals & Ser H56 OG (H2) & 4.1 \\
\hline Heme CA-propionate & van der Waals & TyrH53 (H2) & 3.3 \\
& van der Waals & SerH56 (H2) & 3.9 \\
Heme CD-propionate & H-bond & SerH52 OG (H2) & 2.9 \\
O1D & van der Waals & TyrH53 (H2) & 4.2 \\
& long range ionic & LysL150 NZ (CL) & 3.7 \\
\hline
\end{tabular}

${ }^{1}$ The average of two distances measured for both complexes in the asymmetric unit is listed

${ }^{2} \mathrm{CL}$ : constant light chain. These residues belong to the second molecule in the asymmetric unit or to a symmetric molecule so that their interactions probably result from crystal packing. 


\section{Supplementary data}

\section{Supplementary discussion}

\section{Other modeling trials of the heme domain ·cytochrome $c$ complex.}

The starting structure of the heme $b_{2}$ domain used for the calculation presented in the main text was that in complex with the Fab. In view of its differences with the domain in the 1FCB and 1KBI structures, in particular the heme rotation, we also attempted to model the heme $b_{2} \cdot$ cytochrome $c$ complex using the domain coordinates from the original 1FCB structure. The results (Table S2, Fig. S2B) show that the intermolecular energy and the buried surface area are higher than in the previous model. In addition, we also tested, as active residues, amino acids that lie on the other side of the exposed heme edge, some of which also belong to the interface with the FDH domain (Table S2, Fig. S2B). Again, the complexes in these models are less stable and have a buried surface area that is higher than in the first model. In addition, although the distance between the irons of the two hemes is reasonable (13.3 to $19.6 \AA$ ), a protein loop from cytochrome $c$ separates the two heme groups. 
Table S1: Oligonucleotides used for cloning and sequencing the mouse Fab cDNA

LCNB2B4: 5'-ATG GCC TGG A (CT)T TCA CTT AT-3', consensus mouse $\lambda$ chain leader sequence LCCB2B4 : 5'-GA(AG) ACA (GT)TC TGC A(GC)G AGA CAG ACT CTT-3', mouse $\lambda \mathrm{C}_{\mathrm{L}}$ domain 3 ' terminal sequence

HCNB2B4 : 5'-GAG GT(GT) CAG CT(GT) CAG (GC)AG TC(AT) GGR (GC)C(AT)-3', corresponding to the amino acid sequence (Fig. S1)

HCNHRB2B4 : 5'-TAT GCA AGG CTT ACA ACC ACA-3', class G1 hinge region sequence.

B2B4Ln05: 5'-ACA CTC ACT TGT CGC TCA AG-3' (light chain)

B2B4Ln06: 5'-CTT GAG CGA CAA GTG AGT GT-3' (light chain)

B2B4Ln07: 5'-CTG GGT TGT CTC CAT ACC CTG-3' (light chain)

B2B4Ln08: 5'-CAG GGT ATG GAG ACA ACC CAG-3' (light chain)

B2B4Hc 01 :5'-TGA GCT GCT CAG AGT GTA GAG-3' ( heavy chain)

B2B4Hc02 : 5'-CTC TAC ACT CTG AGC AGC TCA-3' ( heavy chain)

B2B4Hc03 : 5'-ATG GGG TAC ATA AGC TAC GGA-3' ( heavy chain)

B2B4Hc04 :5'-TCC GTA GCT TAT GTA CCC CAT-3' ( heavy chain) 
Table S2: Statistical analysis of the lowest energy structures for the model of the Fcb2 heme domain in complex with iso-1-cytochrome $c$

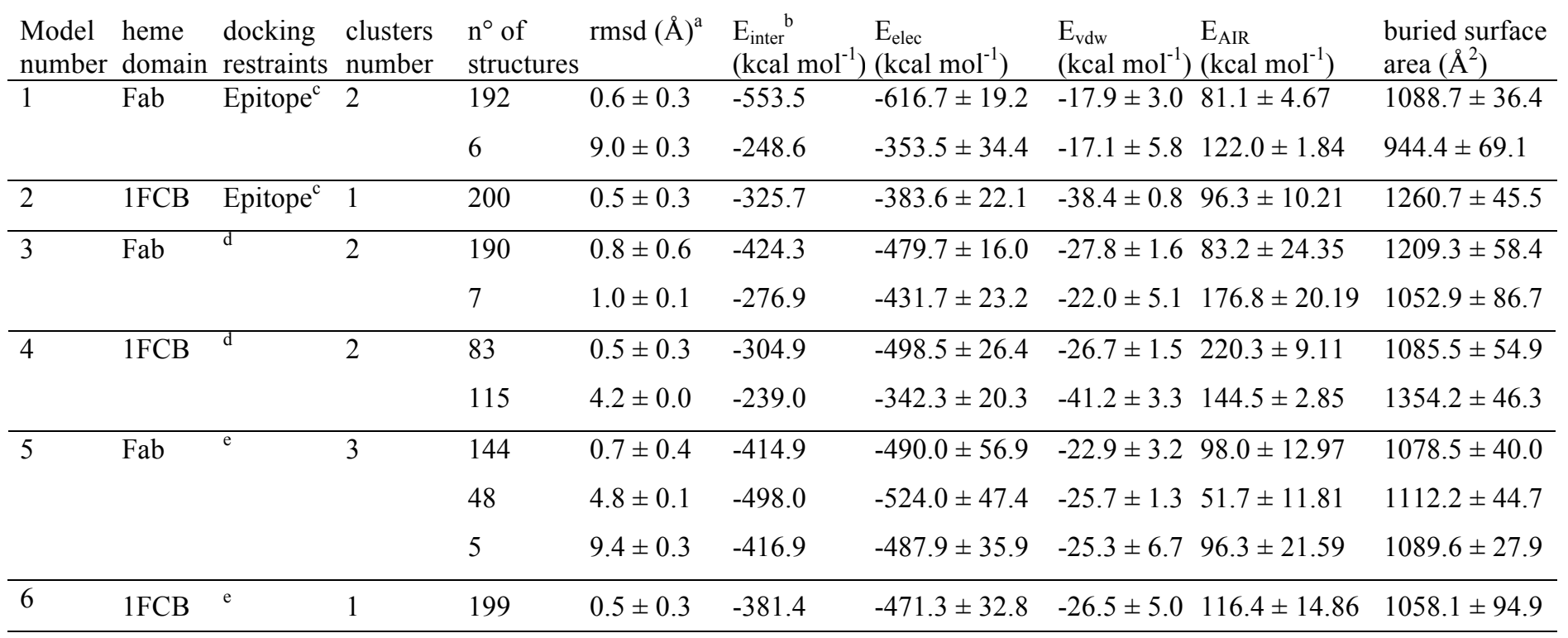

${ }^{\mathrm{a}}$ rmsd from the lowest energy structure

${ }^{\mathrm{b}} \mathrm{E}_{\mathrm{inter}}$ is the intermolecular energy. It is the sum of $\mathrm{E}_{\mathrm{elec}}$ (electrostatic energy), $\mathrm{E}_{\mathrm{vdw}}$ (van der Waals energy), and $\mathrm{E}_{\mathrm{AIR}}$ (energy of the intermolecular restraints).

${ }^{\mathrm{c}}$ Residues $30,63,64,65,67,68,69,70,72$ of the heme domain and the O1A et O1D heme propionate atoms were used as active. Residues 29, 60, 62, 66, 73, 74 and heme $b_{2}$ were used as passive.

${ }^{\mathrm{d}}$ Residues 44, 45, 46, 48, 52, 53, 62, 64, 65, 67, 68, 69 of the heme domain and the O1A et O1D heme propionate atoms were used as active. Residues 47,60, 62, 66 and heme $b_{2}$ were used as passive.

${ }^{\mathrm{e}}$ Residues 39, 44, 63, 65, 68, 69, 72, 73, 74 of the heme domain and the O1A et O1D heme propionate atoms were used as active. Heme $b_{2}$ was used as passive. 


\section{Supplement Figure legend}

Fig. S1: A cDNA and deduced amino acid sequence for the light chain of Fab B2B4. The sequence has been deposited at the EMBL Data Bank (accession number AJ290388) B cDNA and deduced amino acid sequence for the heavy chain $\mathrm{VH}$ and $\mathrm{CH}_{1}$ domains. The sequence has been deposited at the EMBL Data Bank (accession number AJ303449). The underlined sequence was determined at the protein level as described in the text. Numbering of the antibody residues follows the Kabat nomenclature ${ }^{1}$ and the definition of the hypervariable regions is from Chothia et al. ${ }^{2,3}$.

Fig. S2: Models of the Fcb2 heme domain interaction with yeast iso-1-cytochrome $c$ calculated by HADDOCK. A Stereoview of the lowest-energy model of the Fcb2 heme domain (in yellow, Protoheme IX in tan, iron in red) in complex with yeast iso-1-cytochrome $c$ (in blue, heme $c$ in cyan, iron in blue). The residues of the heme domain belonging to the epitope were used as docking restraints (model 1, Table S2). The heme domain is oriented as in Fig. 3B. All the residues from cytochrome $c$ chosen as active in the modeling and all the residues of the heme domain belonging to the epitope (except Asn30) participate in the interactions with cytochrome $c$. In addition, Asn70(c), Lys73(c), Phe82(c) and Gly83(c) are involved in Hbonds and van der Waals contacts with Fcb2. Besides van der Waals and H-bonding interactions, the molecular interface of the complex consists of several salt bridges: between the carboxylates of the heme $b_{2}$ propionate groups and Arg13(c) and Lys87(c), between Glu63( $\left.b_{2}\right)$ and Lys73(c), and between Asp72( $\left.b_{2}\right)$ and Lys72(c). The iron-iron distance is $18.5 \AA$. B Stereo view of the superposition on the heme domain of the lowest-energy solutions of each cluster for the $\mathrm{Fcb} 2$ heme domain cytochrome $c$ complex models analyzed in Table S2. Only the heme domain of model 1 is shown in yellow. Cytochrome $c$ for models 1, 2, 3, 4, 5 and 6 is shown in blue, steel blue, green, tan, magenta and orange, respectively. After superposition on the heme domains, the overall rmsds for $\mathrm{C} \alpha$ atoms of cytochrome $c$ between model 1 and models 2, 3, 4, 5, 6 are 25.5 $\AA, 33.5 \AA, 26.6 \AA, 2.5 \AA, 6.6 \AA$, respectively.

1. Kabat, E., Wu, T. T., Perry, H. M., Gottesman, K. S. \& Foeller, C. (1991). Sequences of proteins of immunological interest (US Department of Health and Human Services, P. H. S., National Institute of Health, Ed.), Bethesda, MD, USA.

2. Chothia, C. \& Lesk, A. M. (1987). Canonical structures for the hypervariable regions of immunoglobulins. J Mol Biol 196, 901-17.

3. Chothia, C., Lesk, A. M., Tramontano, A., Levitt, M., Smith-Gill, S. J., Air, G., Sheriff, S., Padlan, E. A., Davies, D., Tulip, W. R., Colman, P. M., Spinelli, S., Alzari, P. M. \& Poljak, R. J. (1989). Conformations of immunoglobulin hypervariable regions. Nature 342, 877-83. 
Figure. S1

A

1 atg gcc tgg att tca ctt ata ctc tct ctc ctg gct ctc agc tca ggg gcc att tcc cag

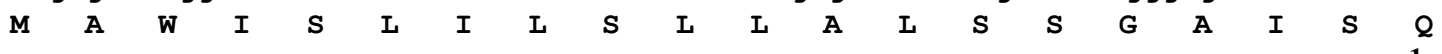

61 gct gtt gtg act cag gaa tct gca ctc acc aca tca cct ggt gaa aca gtc aca ctc act

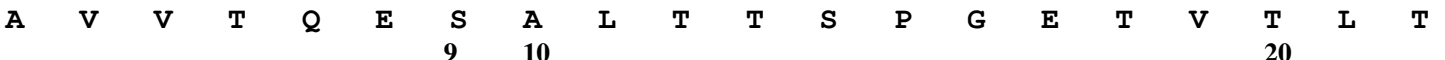

121 tgt cgc tca agt act ggg gct gtt aca act agt aac tat gcc aac tgg gtc caa gaa aaa

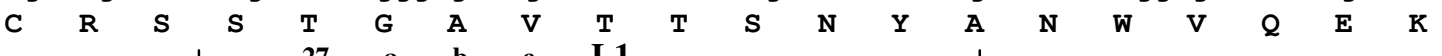

181 cca gat cat tta ttc act ggt cta ata ggt ggt acc aac aaa cga gct cca ggt gtt cct

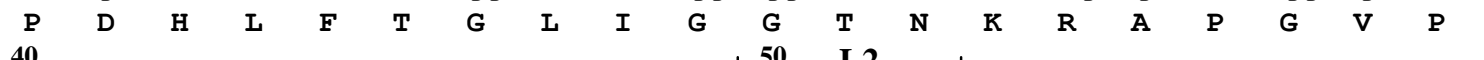

241 gcc aga ttc tca ggc tcc ctg att gga gac aag gct gcc ctc acc atc aca ggg gca cag

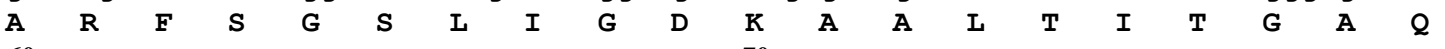
60 70

301 act gag gat gag gca ata tat ttc tgt gct ctg tgg gac agc aac cat ttg gtg ttc ggt

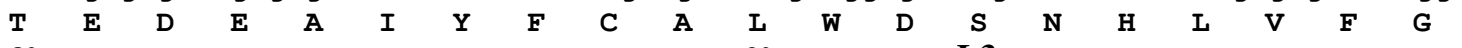
$80 \quad 90 \quad$ L3

361 gga gga acc aaa ctg act gtc cta ggc cag ccc aag tct tcg cca tca gtc acc ctg ttt

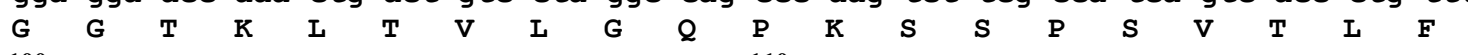
100 110

421 cca cct tcc tct gaa gag ctc gag act aac aag gcc aca ctg gtg tgt acg atc act gat

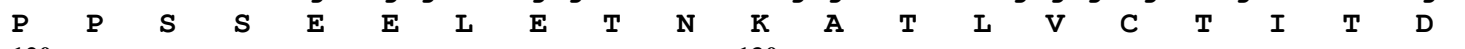
120

481 ttc tac cca ggt gtg gtg aca gtg gac tgg aag gta gat ggt acc cct gtc act cag ggt

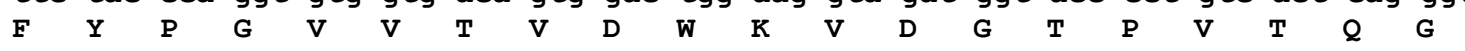
140

541 atg gag aca acc cag cct tcc aaa cag agc aac aac aag tac atg gct agc agc tac ctg

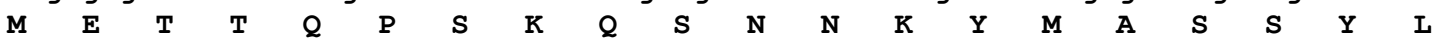
160170

601 acc ctg aca gca aga gca tgg gaa agg cat agc agt tac agc tgc cag gtc act cat gaa

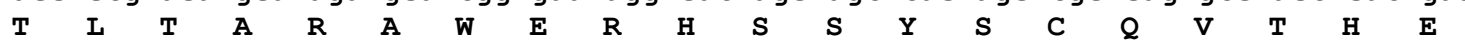
180 190

661 ggt cac act gtg gag aag agt ctg tct cct gca gac tgt

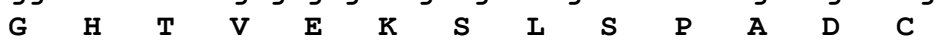
200 
1 gag gtg cag ctt cag gag tca gga cct agc ctc gtg aaa cct tct cag act ctg tcc ctc $\underline{\underline{E}} \quad \underline{V} \quad \underline{Q} \quad \underline{L} \quad \underline{Q} \quad \underline{E} \quad \underline{S} \quad \underline{G} \quad \underline{P} \quad \frac{S}{10} \quad \underline{L} \quad \underline{V} \quad \underline{K} \quad \underline{P} \quad \underline{S} \quad \underline{Q} \quad \underline{T} \quad \underline{L} \quad \underline{S} \quad \frac{L}{20}$

61 acc tgt tct gtc act ggc gac tcc atc acc agt ggt tac tgg aac tgg atc cgg aaa ttc

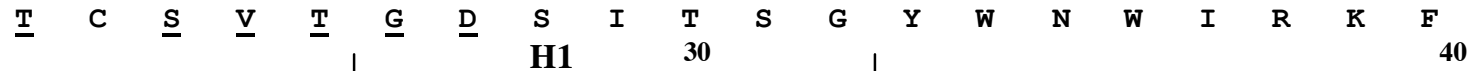

121 cca ggg aat aaa ctt gaa tac atg ggg tac ata agc tac gga ggt agc act tac tac aat $\begin{array}{lllllllllllllllllllll}\mathbf{P} & \mathbf{G} & \mathbf{N} & \mathbf{K} & \mathbf{L} & \mathbf{E} & \mathbf{Y} & \mathbf{M} & \mathbf{G} & \mathbf{Y} & \mathbf{I} & \mathbf{S} & \mathbf{Y} & \mathbf{G} & \mathbf{G} & \mathbf{S} & \mathbf{T} & \mathbf{Y} & \mathbf{Y} & \mathbf{N} \\ & & & & & & & & & & & & \end{array}$

181 cca tct ctc gaa agt cga atc tcc atc act cga gac aca tcc aag aac cag tac tac ctg

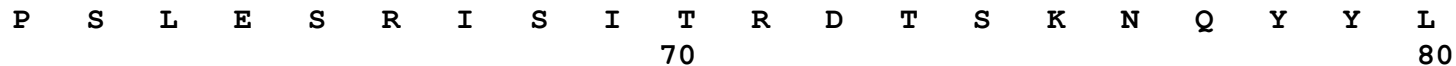

241 cag ttg aat tct gtg act act gag gac aca gcc aca tat ttc tgt gca aga ttg ttt ggt

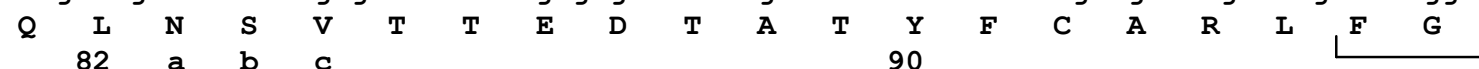

301 tct tac tac ttt gac tac tgg ggc caa ggc acc act ctc aca gtc tcc tca gcc aaa acg $\begin{array}{llllllllllllllllllll}S & Y & Y & F & D & Y & \text { W } & G & Q & G & T & T & \text { L } & \text { T } & \text { V } & \text { S } & \text { S } & \text { A } & \text { K } & T\end{array}$ H3 100 a

361 aca ccc cca tct gtc tat cca ctg gcc cct gga tct gct gcc caa act aac tcc atg gtg $\begin{array}{llllllllllllllllllll}\mathbf{T} & \mathbf{P} & \mathbf{P} & \mathbf{S} & \mathrm{V} & \mathbf{Y} & \mathbf{P} & \mathrm{L} & \mathbf{A} & \mathbf{P} & \mathbf{G} & \mathbf{S} & \mathbf{A} & \mathbf{A} & \mathbf{Q} & \mathbf{T} & \mathbf{N} & \mathbf{S} & \mathbf{M} & \mathbf{V}\end{array}$ 120 130

421 acc ctg gga tgc ctg gtc aag ggc tat ttc cct gag cca gtg aca gtg acc tgg aac tct

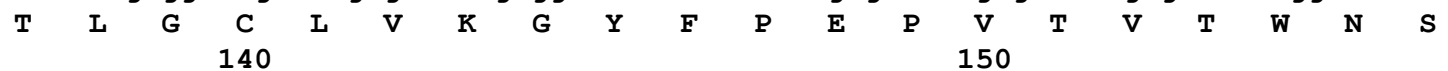

481 gga tcc ctg tcc agc ggt gtg cac acc ttc cca get gtc ctg cag tct gac ctc tac act $\begin{array}{lllcllllllllllllllll}\text { G } & \text { S } & \text { L } & \text { S } & \text { S } & \text { G } & \text { V } & \text { H } & \text { T } & \text { F } & \text { P } & \text { A } & \text { V } & \text { L } & Q & \text { S } & \text { D } & \text { L } & \text { Y } & \text { T }\end{array}$

541 ctg agc agc tca gtg act gtc ccc tcc agc acc tgg ccc agc gag acc gtc acc tgc aac $\begin{array}{llllllllllllllllllll}\text { L } & \text { S } & \text { S } & \text { S } & \text { V } & \text { T } & \text { V } & \text { P } & \text { S } & \text { S } & \text { T } & \text { W } & \text { P } & \text { S } & \text { E } & \text { T } & \text { V } & \text { T } & \text { C } & \text { N }\end{array}$

601 gtt gcc cac ccg gcc agc agc acc aag gtg gac aag aaa att gtg ccc agg gat tgt ggt $\begin{array}{llllllllllllllllllll}\text { V } & \text { A } & \text { H } & \text { P } & \text { A } & \text { S } & \text { S } & \text { T } & \text { K } & \text { V } & \text { D } & \text { K } & \text { K } & \text { I } & \text { V } & \text { P } & \text { R } & \text { D } & \text { C } & G\end{array}$ 200 210

661 tgt aag cct tgc ata

$\begin{array}{lllll}\mathrm{C} & \mathrm{K} & \mathrm{P} & \mathrm{C} & \mathrm{I}\end{array}$ 
Figure S2

A
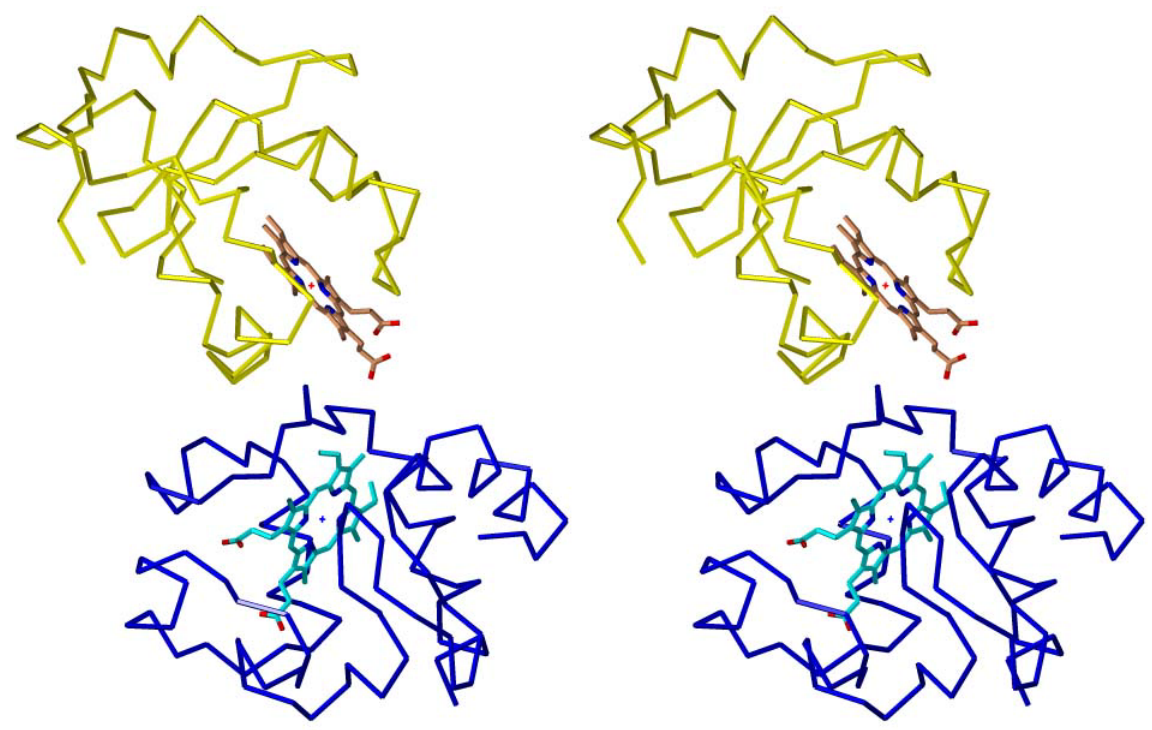

B
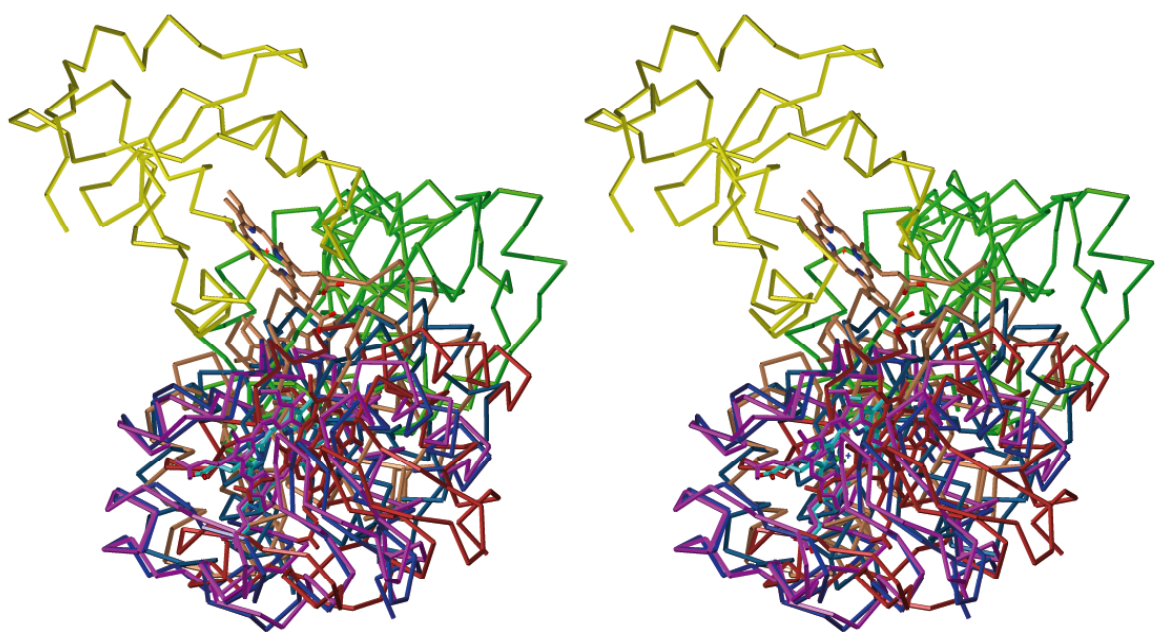